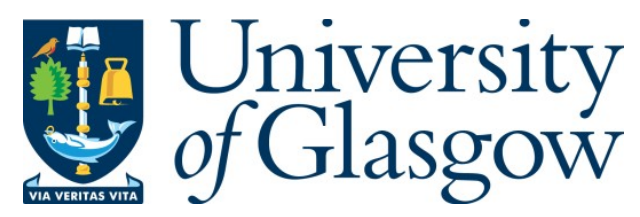

Rahmani, E., Fattahi, A., Karimi, N. and Hosseinalipour, S.M. (2022) A comparative analysis of the evolution of compositional and entropy waves in turbulent channel flows. Physics of Fluids, 34, 017103.

(doi: $\underline{10.1063 / 5.0076350)}$

This is the Author Accepted Manuscript.

This article may be downloaded for personal use only. Any other use requires prior permission of the author and AIP Publishing. This article appeared in Physics of Fluids, 34,017103 and may be found at $(10.1063 / 5.0076350)$.

There may be differences between this version and the published version. You are advised to consult the publisher's version if you wish to cite from it.

http://eprints.gla.ac.uk/259834/

Deposited on: 1 December 2021 


\section{A comparative analysis of the evolution of compositional and entropy waves in turbulent channel flows}

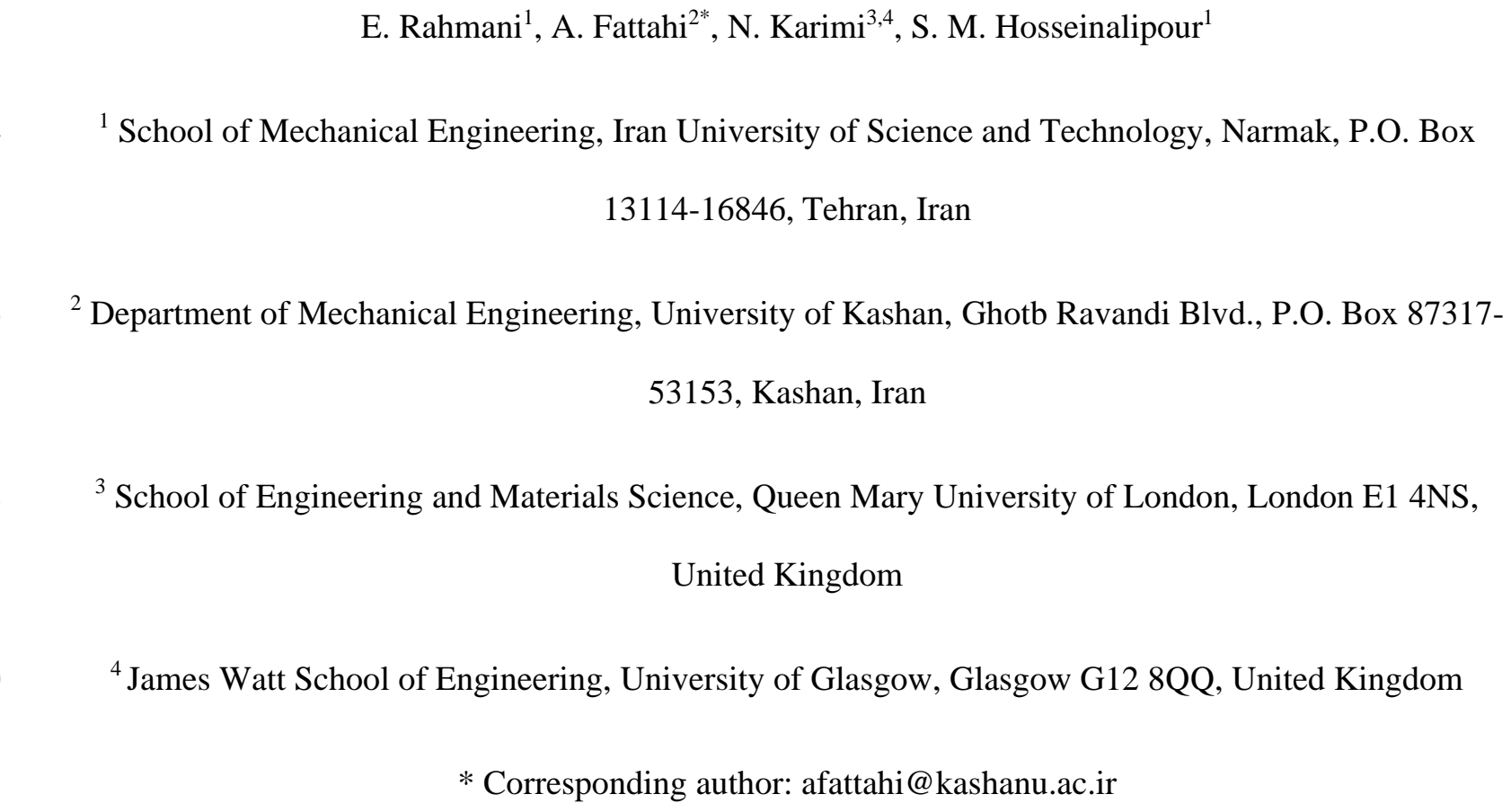

\section{Abstract}

Indirect combustion noise, as an important source of noise in gas turbines, was traditionally attributed solely to entropy waves. In recent years, compositional waves were introduced as another contributor to indirect combustion noise. Nonetheless, unlike that of entropy waves, the annihilation of compositional waves by the mean flow has remained largely unexplored. Hence, the current numerical study analyses the spatiotemporal evolution of different components of compositional waves and compares them with the decay of entropy waves. A convecting wave, including a mixture of combustion products at elevated temperature, is introduced at the inlet of a simple channel. This allows simultaneous analysis of entropy and compositional waves. The passage of these along the channel is modeled using a large eddy simulation and the annihilation of the waves' components is examined in the frequency domain. It is shown that the turbulence level of the mean flow and convective heat transfer on the walls can both result in a considerable wave deterioration. 
1 However, the effects of heat losses from the channel walls are found to be stronger than that of turbulence

2 intensity. Importantly, as the wave is convected, the chemical potential function remains coherent for most

3 of the channel length and deterioration of the compositional wave majorly ensues from the mixture fraction

4 gradient. The results indicate that, overall, the compositional sources feature $10 \%$ to $20 \%$ more dissipation

5 in comparison with the entropic sources. Therefore, compositional waves are less likely to survive the flow

6 and generate noise.

7 Keywords: Entropy waves; Compositional waves; Indirect combustion noise; Coherence analysis; Wave 8 dissipation.

9 1. Introduction

Combustion noise is a major concern associated with the operation of gas turbines and aero-engines

11 for which noise emissions are subject to strict regulations [1]. Further, the reflection of combustion

12 generated noise from the combustor exit nozzle can activate thermoacoustic instabilities and result in

13 significant engine damage $[1,2]$. In general, combustion generated noise is attributed to direct and indirect

14 sources [3]. Unsteady volumetric expansion of the flame, due to heat release, is the origin of the direct noise

15 [4-6]. The indirect noise, however, is emanated from the convecting disturbances of temperature, chemical

16 composition, or vorticity passing through a region of strong pressure gradient and thus flow acceleration

$17[7,8]$. Such disturbances are generated by the flame while their conversion to sound occurs in the combustor

18 exit nozzle and the first stage of the turbine blade [9,10]. It follows that the occurrence of indirect

19 combustion noise depends upon the survival of convecting disturbances throughout their journey from the

20 flame to the downstream nozzles.

Irreversible temperature disturbances are thermodynamically entropy fluctuations convected by the

22 bulk fluid flow and hence they are often called 'entropy waves'. The physics of the conversion of entropy

23 waves to acoustic waves were formally explained by Ffowcs Williams and Howe [11]. Nonetheless,

24 inclusion of entropy waves in aerothermodynamic calculations was initiated in the 1970s by the pioneering 
1 work of Marble and Candel [12]. These authors assumed non-dissipating, one-dimensional entropy waves

2 under the assumption of a compact nozzle and derived analytical expressions for the

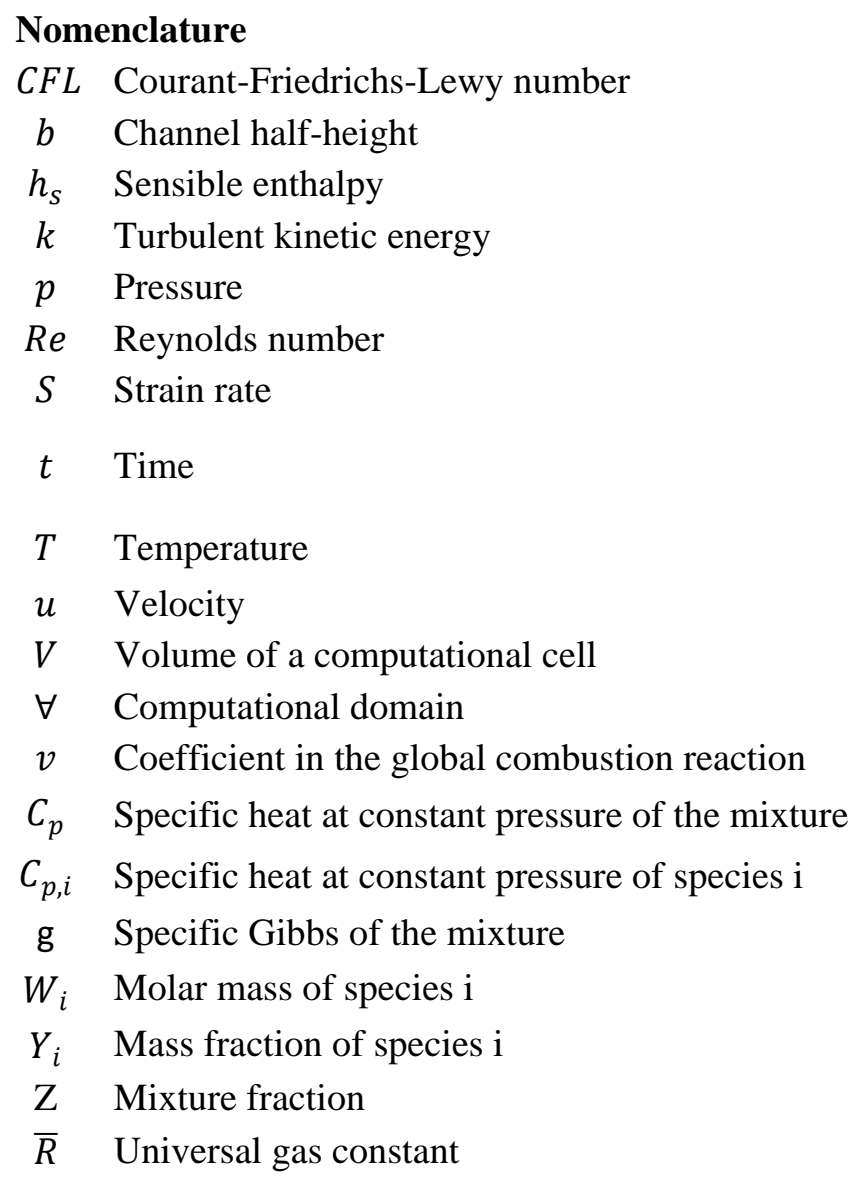

\author{
Greek symbols \\ $\gamma \quad$ Heat capacity ratio \\ $\lambda \quad$ Thermal conductivity \\ $\mu \quad$ Dynamic viscosity \\ $v \quad$ kinematic viscosity \\ $\rho$ density \\ $\tau \quad$ Shear stress \\ $\mu_{i}^{0} \quad$ standard chemical potential of \\ species i \\ $\mu_{i} \quad$ Chemical potential of species i \\ $\Psi \quad$ Chemical potential function
}

$\begin{array}{cl}\text { Subscripts and superscripts } \\ \text { max } & \text { Maximum } \\ \text { res } & \text { Resolved domain } \\ \text { tot } & \text { Total } \\ w & \text { Pertaining to wall } \\ \tau & \text { Pertaining to turbulence } \\ \text { F } & \text { fuel } \\ \text { O } & \text { Oxidizer } \\ \text { i } & \text { ith species }\end{array}$

4 transmitted and reflected noise [12]. More recently, there have been several theoretical studies [13-16],

5 numerical simulations [17-20], and experiments [21-24] aiming to evaluate and release the restrictive

6 assumptions made by Marble and Candle [12]. While these studies advanced the theory considerably, most

7 of them neglected the evolution of entropy waves during their convection by the mean flow. As a result,

8 dissipation and decay of entropy waves received much less attention compared to their conversion to

9 acoustics. This shortcoming led to the issues that are discussed in the followings.

Relevance of entropy waves to the onset of thermoacoustic instabilities was questioned by 11 Sattelmayer and co-workers [25,26], who asserted that entropy waves decay entirely before reaching the 
exit nozzle. These theoretical arguments were also supported by Refs. [27-29]. However, the results of a

2 direct numerical simulation conducted by Morgans et al. [30] were in sharp contrast to those of Sattelmayer

3 et al. [25] and confirmed the survival of entropy waves. Physics of entropy waves indicate that they are of

4 dissipative-dispersive nature [31]. Hence, their conversion to acoustic waves is dominated by the level of

5 dissipation and decay of the wave by the mean flow $[13,32,33]$. The dissipation and dispersion of entropy

6 waves were analyzed by Hosseinalipour et al. [19] and Fattahi et al. [20] through Large Eddy Simulation

7 (LES). These authors considered the dissipative effects of the flow hydrodynamics together with convective

8 heat transfer through the walls of combustor. It was shown that the latter could majorly affect the

9 annihilation of entropy waves and that the inclusion of this effect could explain the reported contradictions

10 in the literature [20].

Recently, Giusti et al. [34] conducted an experimental and numerical study to further explore the

12 decay mechanisms of entropy waves. It was shown that shear dispersion of the mean flow at low Helmholtz

13 numbers and turbulent mixing at large Helmholtz numbers are the key factors deteriorating the wave [35].

14 Ron and Chana [36] compared LES and URANS to identify which one is more appropriate for predicting

15 the evolution of entropy waves. LES allowed prediction of the spatiotemporal evolutions of the wave, while

16 URANS overestimated the temperature level of the hot spots and the resultant noise [36]. Gant et al. [37,38]

17 developed an analytical model to predict the response of a flame to entropic waves, [38] and emphasized

18 the role of turbulent mixing in practical configurations [37]. By conducting LES, Moreau et al. [39] showed

19 that the amplitude of entropy wave subsided and its shape dispersed during convection in a long path. These

20 authors cast doubt on the importance of shear dispersion due to the low frequency of the investigated

21 entropy waves and reported little wave dispersion. This outcome was in keeping with that of Morgans et al.

22 [40], which was later challenged by Fattahi et al. [20] due to the exclusion of thermal dispersion of the

23 wave.

In an experimental study, Wassmer et al. [41,42] showed that entropy wave fluctuations are strongly

25 related to Strouhal number, Reynolds number of the mean flow, and axial location of the wave. They also 
reported the existence of a critical frequency that imparts significant annihilation of the entropy wave. Later,

2 this was confirmed experimentally by Hosseinalipour et al. [43] in their study of entropy wave evolution.

3 In keeping with the findings of Motheau et al. [44], Giusti et al. [35] asserted that deterioration of entropy

4 waves was dominated by mixing through the dilution port of a rich-quench-lean combustor. Bach et al. [45]

5 reported convection in long passages, high frequency, non-uniform acceleration, and shear dispersion as

6 the main reasons for attenuation of entropy waves in the stator of gas turbines, while the film cooling

7 contributed to the wave dissipation in a lower extent [45]. The first two reasons of Bach et al. [45] were

8 also provided by Persico et al. [46], who generated entropy waves by injecting hot/cold flow in a real gas

9 turbine. In their experimental study, Wang et al. [47] declared that entropy waves were vulnerable to heat

10 transfer between the hot plume and cold walls. This was in keeping with the computational findings of

11 Fattahi et al. [20] and Christodoulou et al. [48] who reported a fast decay of entropy waves in heat

12 transferring channels.

Over 40 years ago, Sinai [49] showed theoretically that chemical inhomogeneities can become a source of sound. This has arisen from the perturbations in the chemical composition of the flue gases downstream of the flame. Three decades later, Magri et al. [50,51] formulated this source of indirect noise by using the theory of compact nozzles. Although thermodynamically, chemical inhomogeneities are

17 entropy disturbances, the noise generated by them is often termed 'compositional noise' and is distinguished

18 from entropy noise produced by hot spots. Magri et al. [50] showed that for a lean premixed combustor 19 with a supersonic exit nozzle, the compositional noise could exceed entropy noise. They highlighted the 20 importance of dissipation of compositional wave, although no analysis on this subject was reported. In an 21 experimental effort, Rolland et al. [22] investigated entropic and compositional waves, and argued that 22 dispersion and diffusion had a minor effect on the waves' deformation. By extending an earlier model 23 presented by Domenico et al. [52], it was declared that the compact-nozzle theory might overestimate the 24 compositional noise [53]. They showed that the assumption of an isentropic nozzle could lead to inaccurate 25 predictions and indicated that the compositional perturbation might experience minimal dispersion in short 
tubes. Using URANS modeling and by injecting the inert gases, Rodrigues et al. [54] argued that the

2 dispersion effects on the compositional waves remain insignificant.

The proceeding review of the literature clearly shows that dissipation and dispersion of entropy waves are essential parts of indirect noise generation. Recent studies on the spatiotemporal evolution of entropy waves have revealed the role of complex hydrodynamic and thermal effects in the decay of entropy waves and the subsequent sound generation $[19,20,43,55,56]$. However, so far, there is no systematic investigation focused on the decay of compositional waves. Existing studies of compositional noise have speculated rather differently about the evolution of these waves [22,57-59] and a conclusive view is yet to emerge. The current work aims to address this issue through conduction of high fidelity numerical simulations of the propagation process of a compositional wave in turbulent channel flows.

\section{Problem configuration, boundary, and initial conditions}

The investigated configuration consists of two parallel plates shown in Fig. 1a. The geometry and dimensions are the same as those of Ref. [30], assuming $b=2.5 \mathrm{~mm}$. This simple geometry allows for exploring the physics of wave annihilation without intervention of the excess parameters. The base fluid flow is air under atmospheric pressure, temperature of $300 \mathrm{~K}$, and is treated as an ideal gas. Periodic boundary conditions are applied on the lateral and stream-wise directions of the channel. The zero axial gradient for all properties is set at the channel outlet. The turbulent Reynolds number is considered as 180, similar to that considered by Morgans et al. [30] in a similar configuration. A steady, fully-developed velocity profile is introduced at the channel inlet, corresponding to Reynolds number of 13600, similar to those earlier used for entropy waves in numerical simulations $[19,20,40]$. The inlet flow properties remain constant except for the duration that the entropic-compositional wave is introduced. The convective heat transfer on the external upper and bottom walls is set by implementing the thermal convective coefficient of $100 \mathrm{~W} / \mathrm{m}^{2} . \mathrm{K}[60]$ and the ambient temperature of $273 \mathrm{~K}$. The walls are characterized by zero thickness and no-slip velocity conditions. Reynolds number is defined based on the bulk flow velocity, $U_{b}=$ $20.94 \mathrm{~m} / \mathrm{s}$, and the hydraulic diameter of the channel, $D_{h} \cong 10 \mathrm{~mm}$. Fig.1b demonstrates the distribution 
1 of the probes on the channel cross-section that are later used for the analysis of the wave's surface

2 destruction. They were chosen to detect the wave in the central and bilateral zones by recording data such

3 as temperature and species fractions. To limit the volume of data recording, only five points were selected.

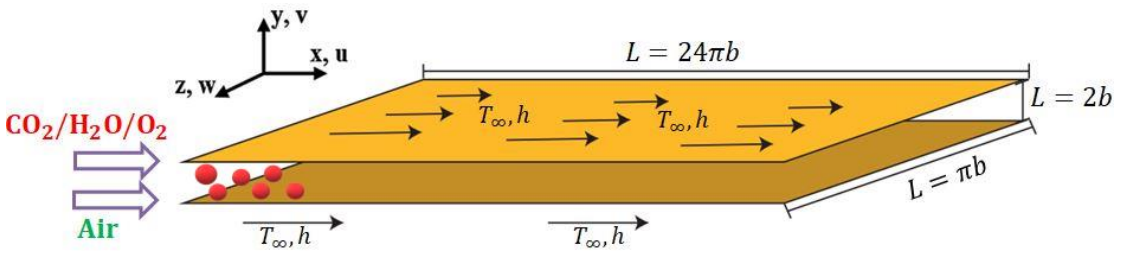

(a)

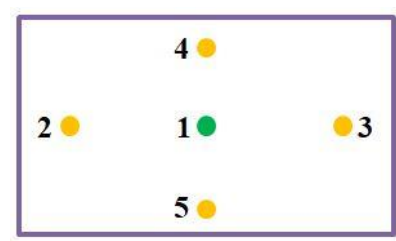

(b)

Fig. 1. (a) Schematics of the problem configuration, and (b) the distribution of the probes on the wave's surface.

4

To generate entropic-compositional waves, a parcel of hot fluid with different chemical composition compared to the base flow is injected uniformly into the whole inlet cross-section. This blob of gas is comprised of $\mathrm{H}_{2} \mathrm{O}, \mathrm{CO}_{2}$ and $\mathrm{O}_{2}$ as the products of complete combustion of n-dodecane (Kerosene substitute) in pure oxygen at an equivalence ratio of 0.8 . This mixture is injected at the temperature of $330 \mathrm{~K}$ for the duration of $0.000545 \mathrm{~s}$ such that $0.093 \mathrm{~kg} / \mathrm{s}$ of gas is added to the main flow. The injection includes a linear rising, a constant while, and an exponential dropping (see Ref. [19] for further details). Upon injection, the generated pulse is convected throughout the channel by the base fluid flow. It is noted that this pulse features a different temperature and chemical composition compared to the bulk fluid flow and therefore both entropy and compositional waves have been generated.

\section{Numerical and theoretical methods}

\subsection{Governing equations and numerical procedure}

Entropy and compositional waves can be affected by turbulent mixing [37], heat transfer [20], and diffusion [42]. Hence, a high fidelity numerical approach is required to capture the spatiotemporal evolution of the wave during the advection process in the channel. For this purpose, transient LES is a reasonably precise and affordable numerical approach [36] and is therefore utilized in this work. 
In LES, all the equations are derived by filtering on the physical domain due to the separation of

2 large and small-scale eddies. The large-scale decomposition of the general property of $\varphi$ can be obtained

3 by

$$
\bar{\varphi}(x)=\int_{V} \varphi\left(x^{\prime}\right) G\left(x, x^{\prime}\right) d x^{\prime}, x^{\prime} \in V
$$

$$
\begin{aligned}
& \frac{\partial \bar{\rho}}{\partial t}+\frac{\partial}{\partial x_{j}}\left(\bar{\rho} \tilde{u}_{j}\right)=0, \\
& \frac{\partial}{\partial t}\left(\bar{\rho} \tilde{u}_{i}\right)+\frac{\partial}{\partial x_{j}}\left(\bar{\rho} \tilde{u}_{i} \tilde{u}_{j}\right)=-\frac{\partial \bar{P}}{\partial x_{i}}+\frac{\partial}{\partial x_{j}}\left(\bar{\tau}_{i j}-\bar{\rho}\left(\widetilde{u_{\imath} u_{j}}-\tilde{u}_{i} \tilde{u}_{j}\right)\right),
\end{aligned}
$$

7 whereby $\rho, u$ and $p$ are fluid density, velocity, and pressure, respectively. Also, symbols ${ }^{-}$and $\sim$ denote 8 spatial and Favor filtering, related to each other by $\tilde{\varphi}=\bar{\rho} \bar{\varphi} / \bar{\rho}$, in which $\varphi$ is an arbitrary variable [61]. In

9 Eq. (3), $\bar{\tau}_{i j}$, computed directly in the domain as the large scale, is obtained by

$$
\tau_{i j}=\rho \overline{u_{\imath} \bar{u}_{j}}-\rho \bar{u}_{i} \bar{u}_{j}
$$

10 The subgrid-scale (SGS) stress tensor, $\tau_{i j}^{S G S}$, which requires finer grid size and a model to calculate [62], is

11 determined through

$$
\tau_{i j}^{S G S}-\frac{1}{3} \delta_{i j} \tau_{k k}^{S G S}=-2 \mu_{S G S}\left(\tilde{S}_{i j}-\frac{1}{3} \delta_{i j} \tilde{S}_{k k}\right)
$$

12 where $\mu_{S G S}$ means the subgrid-scale viscosity. The strain rate tensor for the resolved scales, $\tilde{S}_{i j}$, is expressed 13 by

$$
\tilde{S}_{i j}=\frac{1}{2}\left(\frac{\partial \widetilde{u}_{j}}{\partial x_{i}}+\frac{\partial \widetilde{u}_{i}}{\partial x_{j}}\right)
$$

In Eq. (5), $\tau_{k k}^{S G S}$ denotes the isentropic part of the SGS stress tensor written as 


$$
\tau_{k k}^{S G S}=\bar{\rho}\left(\widetilde{u_{k} u_{k}}-\tilde{u}_{k} \tilde{u}_{k}\right)=2 \bar{\rho} k_{S G S}
$$

1 in which $k_{S G S}$ is the SGS kinetic energy. Adapting Local Eddy-viscosity (WALE) [61] and Smagorinsky-Lilly [63] were presented for this purpose.

4 Previous studies showed that WALE is advantageous in confined flows by considering the zero turbulent

5 viscosity for laminar shear layers in comparison to the other models [63]. Hence, WALE model is used in 6 the current study. This model has been also successfully used in LES of entropy waves propagation $7 \quad[19,20,36]$. WALE model calculates $\mu_{t}$ through the following relation

$$
\mu_{t}=\rho L_{s}^{2} \frac{\left(S_{i j}^{d} S_{i j}^{d}\right)^{3 / 2}}{\left(\bar{S}_{i j} \bar{S}_{i j}\right)^{5 / 2}+\left(S_{i j}^{d} S_{i j}^{d}\right)^{5 / 4}}
$$

$8 \quad$ where $S_{i j}^{d}=\frac{1}{2}\left(\bar{g}_{i j}^{2}+\bar{g}_{j i}^{2}\right)-\frac{1}{3} \delta_{i j} \bar{g}_{k k}^{2}$ and $\bar{g}_{i j}=\frac{\partial \bar{u}_{i}}{\partial x_{j}} . L_{s}$ is a function determining the mixing length of the $9 \quad$ subgrid-scale as [61]

$$
L_{S}=\min \left(\kappa d, C_{w} V^{1 / 3}\right) \text {, }
$$

in which $\kappa$ is von-Karman's constant, set to be 0.4 in the current simulation (following Ref. [61] ) and $d$ is

11 the minimum distance of each computational cell from the wall. Applying LES filters to the energy equation 12 renders

$$
\frac{\partial}{\partial t}\left(\bar{\rho} \tilde{h}_{s}\right)+\frac{\partial}{\partial x_{j}}\left(\bar{\rho} \tilde{u}_{j} \tilde{h}_{s}\right)=\frac{D \bar{P}}{D t}+\bar{\tau}_{i j}: \frac{\partial \widetilde{u}_{j}}{\partial x_{i}}+\frac{\partial}{\partial x_{j}}\left(\frac{\mu}{P r} \frac{\partial \widetilde{h}_{s}}{\partial x_{j}}-\bar{\rho}\left(\widetilde{u_{j} h_{s}}-\tilde{u}_{j} \tilde{h}_{s}\right)\right)
$$

13 in which $\bar{h}_{S}$ and $P r_{S G S}$ are the sensible enthalpy and SGS Prandtl number, correspondingly. The term $14 \bar{\rho}\left(\widetilde{u_{j} h_{s}}-\tilde{u}_{j} \tilde{h}_{s}\right)$ on R.H.S of Eq. (10) is the SGS enthalpy flux and is determined by

$$
\bar{\rho}\left(\widetilde{u_{j} h_{s}}-\tilde{u}_{j} \widetilde{h}_{s}\right)=-\frac{\mu_{S G S}}{P r_{S G S}} \frac{\partial \widetilde{h}_{S}}{\partial x_{j}}
$$

The filtered equation for species transport reads 


$$
\left.\frac{\partial}{\partial t}\left(\bar{\rho} \tilde{Y}_{k}\right)+\frac{\partial}{\partial x_{j}}\left(\bar{\rho} \tilde{u}_{j} \tilde{Y}_{k}\right)=\frac{\partial}{\partial x_{j}}\left(\bar{\rho} \widetilde{D}_{k}+\frac{\mu_{t}}{S c_{t}}\right) \frac{\partial \tilde{Y}_{k}}{\partial x_{j}}\right)
$$

1 where $Y_{k}$ and $D_{k}$ are respectively the mass fraction and mass diffusivity of $i$ th species and $S c_{t}$ shows the

2 turbulent Schmidt number. To involve compressibility effects, the ideal gas law is included in the governing

3 equations.

The finite volume method in conjugation with the coupled algorithm, which solves the equations of

5

$$
C F L=u_{1 \max } \Delta t / \Delta x_{\min }
$$

8 became less than unity [64]. CFL involves the highest bulk velocity $\left(u_{1 \text { max }}\right)$ and minimum grid size

$9\left(\Delta x_{\min }\right)$. For the simulations reported here, $C F L=0.28$. Since there is no acceleration in the investigated
low-Mach number flow, the conversion of entropy and compositional waves to acoustic waves does not happen. The acoustic waves are, therefore, excluded from the analysis, and the bulk flow time-step is used in the $C F L$ number calculation.

The initialization data feed the solution process and the fully-developed velocity at the inlet was obtained by a steady RANS model. A while equal to triple of the time needed for the advection of a massless fluid element from the channel inlet to the outlet was elapsed for washing out and reaching the stationary condition.

\subsection{The sources of indirect noise}

Magri et al. [65] derived the following equation which relates entropy fluctuations to those of temperature, pressure, and mass fraction of chemical species.

$$
\frac{d s}{C_{p}}=\frac{d T}{T}+\frac{c_{p}{ }^{\prime}}{c_{p}} d Z-\Psi d Z-\frac{\gamma-1}{\gamma} \frac{d p}{p}
$$


1 where $\Psi$ denotes the chemical potential, $c_{p}$ is the specific thermal capacity of the mixture and prime

2 indicates the derivative with respect to the mixture fraction, $Z$, which relates to the mass fraction of each

3 component, $\mathrm{Y}_{i}$, by $[66,67]$

$$
Y_{i}=Y_{i O, 0}+\left(Y_{i F, 0}-Y_{i O, 0}\right) Z-\frac{W_{i} v_{i}}{W_{F} v_{F}}\left(Y_{F}-Y_{F, 0} Z\right)
$$

4

5

6

7

$$
\Psi=\frac{1}{c_{p} T} \sum_{i}\left(\mu_{i} / W_{i}\right) Y_{i}^{\prime}
$$

8 in which $\mu_{i}$ is the chemical potential of $i$ th component, given by $[65,68]$

$$
\mu_{i}=\mu_{i}^{0}+\bar{R} T \ln \left(P_{i}\right)
$$
17 (14) are named $s_{1}$ to $s_{4}$.

In Eq. (17), superscript 0 denotes standard conditions, while $\bar{R}$ and $P_{i}$ represent the universal gas constant and the partial pressure of each gaseous component in the mixture, respectively. It is worth noting that $Y_{i}^{\prime}$ in Eq. (16) can be readily found from Eq. (15). Further, $c_{p}=\sum_{i} c_{p, i} Y_{i}$, which is for a mixture of ideal gases, and the term $c_{p}{ }^{\prime}$ can be calculated using Eq. (15).

Each term on R.H.S of Eq. (14) shows a source of indirect noise. The first term represents the hot spot advecting throughout the channel, as the conventional source of indirect noise. The next two terms are the compositional sources and the last term is the pressure fluctuations included in entropy fluctuations. In order to analyze the evolutions of these sources, the absolute value of the terms appearing on R.H.S of Eq.

\subsection{Grid independency and validation}


To ensure the grid independency of the simulations, various grid sizes were investigated considering

2 the area below the temperature-time graph of the convecting wave. This revealed that a grid consisting of

3500000 cells could be fine enough to capture the investigated physics. The grid was shaped non-uniformly

4 in $y$ direction to capture the near-wall region properly, while the cells in other directions were formed

5 uniformly. However, since LES is rather sensitive to grid size, $L E S_{I Q_{k}}$ index [69] was also checked for

6 various grid sizes. This index is defined as:

$$
L E S_{-} I Q_{k}=\frac{k^{r e s}}{k^{t o t}}
$$

7 in which $k^{r e s}$ denotes the resolved kinetic energy and $k^{\text {tot }}$ shows the total kinetic energy. This index can

8 be used to qualify whether the LES grid senses the sub-grid scale or not. It was shown that this index should

9 be higher than 0.75 for fully turbulent flow conditions [70]. It was also found that $L E S_{-} I Q_{k}$ index in this

10 simulation was about 0.91 , assuring quality of the utilized grid. The finer grid near the walls was evaluated

11 by a non-dimensional distance, $y^{+}$. In this study, $y^{+}$is about 0.9 , as the values below unity were

12 recommended by LES references [71,72]. Fig. 2a shows a comparison between the non-dimensional

13 velocity, $U^{+}$, versus $y^{+}$for the current study and the DNS results of Moser et al. [73]. Here, $U^{+}$and $y^{+}$are

14 defined as follows.

$$
U^{+}=\frac{U}{u_{\tau}}, y^{+}=\frac{\rho u_{\tau} \Delta y}{\mu}
$$

15 where $\Delta y$ and $U$ depict respectively the distance of the cell from the nearest wall and the bulk flow velocity.

16 For further comparison, the root-mean-square of the velocity components $\left(u^{\prime+}, v^{\prime+}, w^{\prime+}\right)$ versus $y^{+}$is

17 illustrated in Fig. 2b. The negligible discrepancy (lower than 2\%) in this figure shows that the simulation

18 can be almost as precise as a DNS. In order to show the ability of the current simulations to capture the

19 physics of convecting thermal fluctuations, the simulation results were compared to the data from the

20 experiments of Bake et al. [23], see Fig. 2c. The difference found in the rear of the entropy wave could be

21 attributed to the unknown location of the thermocouple in Ref. [23]. This is why the mass-weighted average

22 was used to calculate the temperature fluctuations. Figure $2 \mathrm{c}$ shows a high level of similarity between the 
1 two sets of data. The current results for species distribution influenced by the advection and diffusion are

2 also compared to the experimental data of Shavit and Lavan [74] in which mixing of two jets of air and

3 argon was investigated. Fig. 2d shows this comparison at the distance of 7.5 inches downstream of the jet

4 inlet. Given the 7\% average disparity, once again, the comparison shows a good agreement between the

5 experimental data and simulation results and demonstrates the suitability of the current numerical setting.

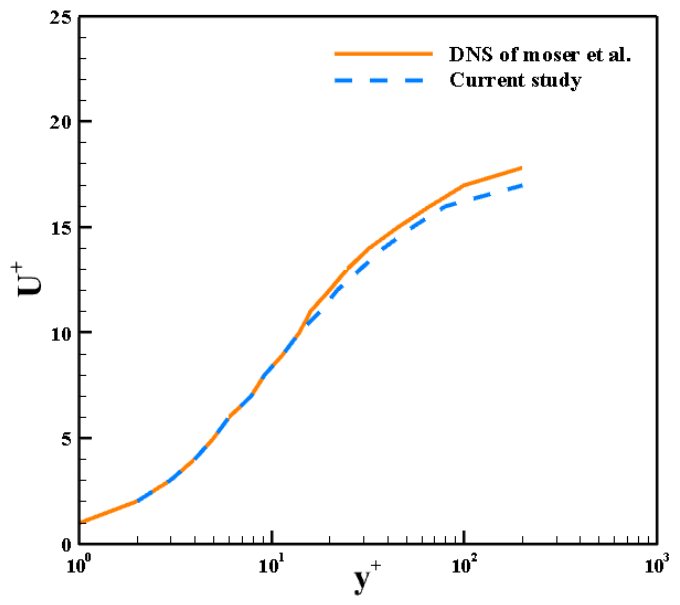

(a)

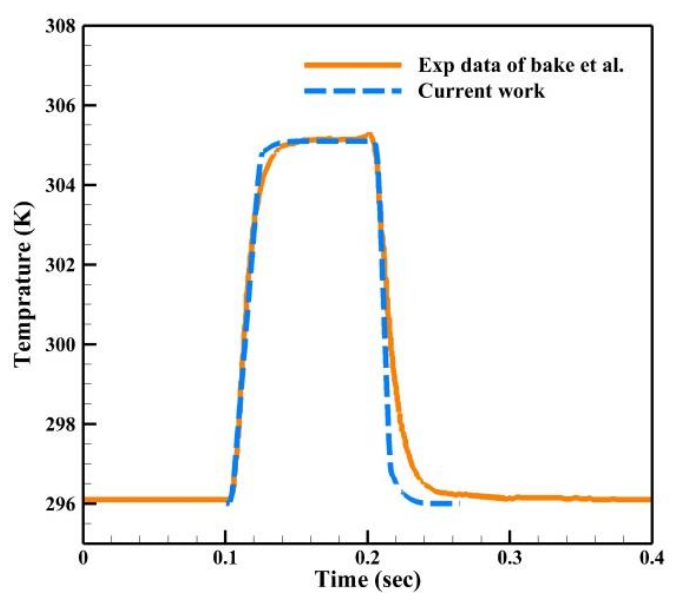

(c)

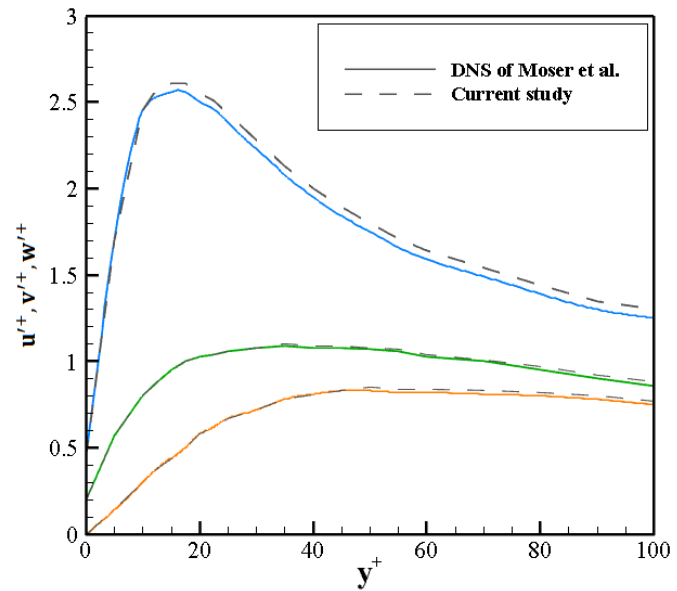

(b)

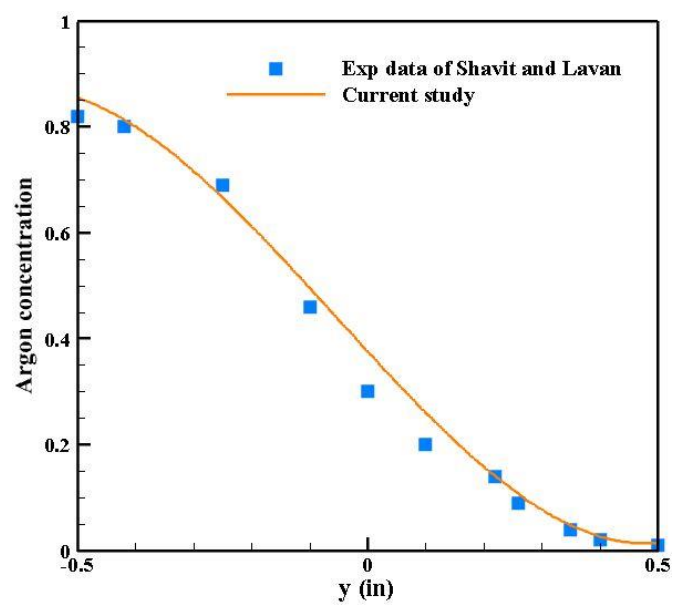

(d)

Fig. 2. A comparison between the current LES results and (a) the DNS of Moser et al. [73] for $\mathrm{U}^{+}$, (b) $\mathrm{u}^{\prime+}$ (the upper graph), and $\mathrm{w}^{1+}$ (the lower graph) as well as experiments of (c) Bake et al. [23], and (d) Shavit and Lavan 
In the current simulations, it is essential to ensure that the non-physical numerical dispersion of the

2 wave is negligibly small and thus the observed dispersion is entirely physical. Although significant attempts

3 were made to subside the numerical diffusion by choosing a fine and structured grid, the extent of numerical

4 diffusion should be evaluated. To do so, an Eulerian flow with zero thermal conductivity and viscosity was

5 set up in the channel with similar other parameters compared to the main simulations. A temperature pulse

6 was set in the channel and the mass-weighted average of the temperature increment divided by the base

7 flow temperature was detected at various longitudinal positions over the cross-section of the channel, see

8 Fig. 3. Evidently, the wave distortion is very small. Calculation of the area under the wave in this figure

9 showed about 3\%difference between the wave at the inlet and outlet. This indicates that the current simulation is free from any considerable numerical diffusion and the observed dispersion is physical.

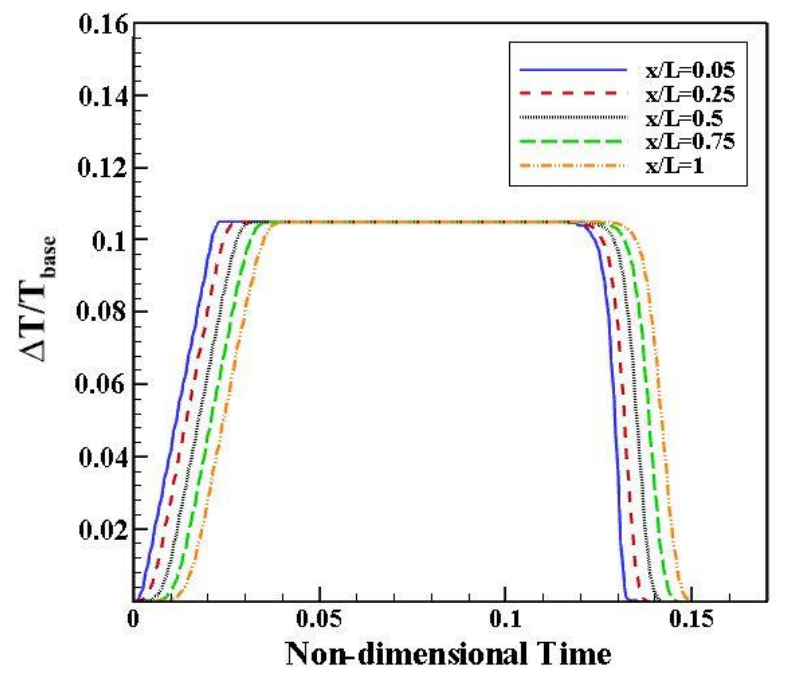

Fig. 3. The temperature pulse in various longitudinal locations in an Eulerian flow simulation.

\section{3.4. Coherency of the wave, wave specification, and dissipation}

Coherence function is a measure determining how much a chosen pair of signals are linearly related.

14 This measure is provided in the frequency domain and the capital letters in the subsequent relations denote 15 spectral quantities. The coherence function takes a numerical value between zero and unity. The former 16 shows fully unrelated and the latter denotes completely linearly related signals. This function has been also 
1 used in previous studies $[19,20,75]$ and has demonstrated its ability to capture wave deterioration. In

2 general, for the two signals of $x(t)$ and $y(t)$, coherence is defined in the frequency domain as

$$
C_{x y}(f)=\frac{\left(G_{x y}(f)\right)^{2}}{G_{x x}(f) G_{y y}(f)}
$$

3 whereby $G_{x x}(f)$ and $G_{y y}(f)$ mean the auto-spectral density of the preceding signals. The auto and cross-

4 spectral density are calculated using the mathematical expectation function, $E(-)$, as follows.

$$
\begin{aligned}
& G_{x x}(f)=E[X(\omega) X(\omega)], \\
& G_{x y}(f)=E[X(\omega) Y(\omega)] .
\end{aligned}
$$

Further details can be found in Ref. [76]. In this study, the coherence function is used for measuring the relation of a wave between two distinct spatial locations. It is performed using the mass-weighted

7 average of the source value, $\bar{\varphi}$, on the wave's front between the spatial locations of $x_{i}$ and $x_{j}$, as

$$
\operatorname{Coh}_{L}=\text { Coherence }\left[\bar{\varphi}\left(x_{i}\right), \bar{\varphi}\left(x_{j}\right)\right] \text {. }
$$

8 Further, to evaluate the wave's frontal destruction, the coherence is calculated between the central 9 probe value, labeled 1 in Fig. $1 \mathrm{~b}$ and shown by $\varphi\left(\mathrm{p}_{1}\right)$ and the mass-weighted average of other lateral points, 10 meaning $\bar{\varphi}\left(\mathrm{p}_{2}-\mathrm{p}_{5}\right)$, which reads

$$
\operatorname{Coh}_{D}=\text { Coherence }\left[\varphi\left(\mathrm{p}_{1}\right), \bar{\varphi}\left(\mathrm{p}_{2}-\mathrm{p}_{5}\right)\right]
$$

$$
S t=\frac{f(H z)}{\frac{U_{b}}{L}}
$$

13 in which $f$ is the frequency, $U_{b}$ expresses the bulk flow velocity and $L$ indicates the channel length. It has

14 been shown experimentally that the channel length is the correct length scale to be included in the definition 15 of the Strouhal number [43]. 
To analyze the decay processes, delineation of the wave rear and front is of importance. The diffusion

2 and mixing mechanisms may smear the wave boundaries. Therefore, a criterion similar to that employed in

3 the previous works $[19,20]$ is used here to unambiguously define the wave location. By setting the criterion

4 value (R.H.S) of the following relation to 0.02 and 0.001 , the rear and front of the wave are correspondingly

5 detected.

$\frac{\bar{\varphi}-\bar{\varphi}_{i}}{\bar{\varphi}_{i}}=$ criterion value

6 in which $\bar{\varphi}$ is either temperature or species concentration derived by mass-weighted average. Further, $i$

7 stands for the value of $\bar{\varphi}$ before releasing the pocket of gaseous mixture.

8 The thermal and chemical waves, as considered in this work, both experience dissipation and decline 9 of the wave's energy [20,77]. The dissipation denotes how much the wave's thermal energy or chemical 10 uniformity is affected by the fluid flow. Therefore, the definition of dissipation involves the amplitude 11 decline of the sources in the frequency domain, determining the wave annihilation. Dissipation can be 12 quantitatively determined by the following equation $[14,19,43]$.

$$
\text { Dissipation }\left(\mathrm{f}_{\mathrm{a}}\right)=\left.\frac{\mathcal{F}\left[\left(\left.\bar{\varphi}(\mathrm{t})\right|_{\mathrm{x}_{\text {inlet }}}\right)\right]-\mathcal{F}\left[\left(\left.\bar{\varphi}(\mathrm{t})\right|_{\mathrm{x}_{\text {outlet }}}\right)\right]}{\mathcal{F}\left[\left(\left.\bar{\varphi}(\mathrm{t})\right|_{\mathrm{x}_{\text {inlet }}}\right)\right]}\right|_{\mathrm{f}=\mathrm{f}_{\mathrm{a}}}
$$

13 where $\mathcal{F}$ shows the Fourier transformation and $\bar{\varphi}(\mathrm{t})$ denotes the arithmetic average of the sources value,

14 in the arbitrary frequency of $f_{a}$.

\section{4. Results and discussion}

As stated earlier, it is assumed that the combustion products of n-dodecane are convecting through

17 the duct. The current study investigates the evolution of sources of indirect noise, which are $s_{1}=\frac{d T}{T}, s_{2}=$ $18\left|\frac{C_{p}{ }^{\prime}}{C_{p}} d Z\right|, s_{3}=|-\Psi d Z|$, and $s_{4}=\left|-\frac{\gamma-1}{\gamma} \frac{d p}{p}\right|$ in the channel shown in Fig. 1. The last source, $s_{4}$, is not 19 considered in this study, due to the extremely small pressure variations caused by the introduction of the 20 waves at the channel inlet. The channel wall is either adiabatic or convectively cooled (see Fig. 1). To 
1 evaluate the effects of turbulence on the waves, two different turbulence intensities are considered. Table 1

2 shows the characteristics of the cases investigated in this work. The high level of turbulence intensity was

3 chosen to show clearly the effects of different turbulence intensities on the investigated sources. In keeping

4 with the literature, the thermal part of the wave (see Eq. (14)) is called entropy wave clarifying that this

5 part is only ensuing from the temperature increment.

Table1- The case descriptions of the current study.

\begin{tabular}{lll}
\hline Case No. & Wall conditions & Turbulence intensity (\%) \\
\hline 1 & Adiabatic & 5 \\
\hline 2 & Adiabatic & 20 \\
\hline 3 & convective & 5 \\
\hline 4 & convective & 20 \\
\hline
\end{tabular}

7

8

9

10

11

12

13

14

15

16

17 that, as expected, during the advection process, the wave amplitude decreases, while the width increases.

18 This is evidence for the wave dissipation and deformation, resulted from turbulence mixing and diffusion

$19[35,53]$. The strongest elongation of the waves is observed in the first half of the channel, while the

20

Figure 4 shows the time trace of temperature increment divided by the base flow temperature as well as the mass fraction of the injected gases. This is shown for all cases investigated at the inlet, middle, and near the outlet. The last location was chosen to avoid unphysical estimations due to the effects of boundary conditions on the waves. The graphs are derived by the mass-weighed average on the whole cross-section.

Time is further non-dimensionalized using the convection time of the waves by the constant bulk velocity along the channel length, that is $L / U_{b}$. The temperature and mass fractions of the injected hot packages of chemical species of carbon dioxide, oxygen, and steam are detected over time in various spatial locations, especially at the inlet, middle, and near the outlet. Temperature pulse denotes the entropic wave and the mass fraction of species is representative of the compositional wave during advection. These traces show amplitude decrement gradually continues until the outlet. The amplitude of the thermally cooled cases 3 
1 and 4 is slightly lower than that of the adiabatic cases of 1 and 2, whilst the width shows the inverse trend.

2 This shows the deteriorating effect of the external walls by cooling the entropy wave, similar to that done

3 by the liner of the gas turbine combustor. Interestingly, the peak value of the traces is almost the same for

4 all investigated cases, as the traces are often similarly affected by the annihilating mechanisms. Convective

5 cooling makes the traces' peak temporally shifted when they are traveling in the second half of the channel.

6 This indicates a strong decay of the wave mainly attributed to thermal and hydrodynamic effects, both

7 occurring in real gas turbine combustors [78]. The turbulence intensity appears to dominate the traces

8 amplitudes by increasing the level of mixing.

inlet
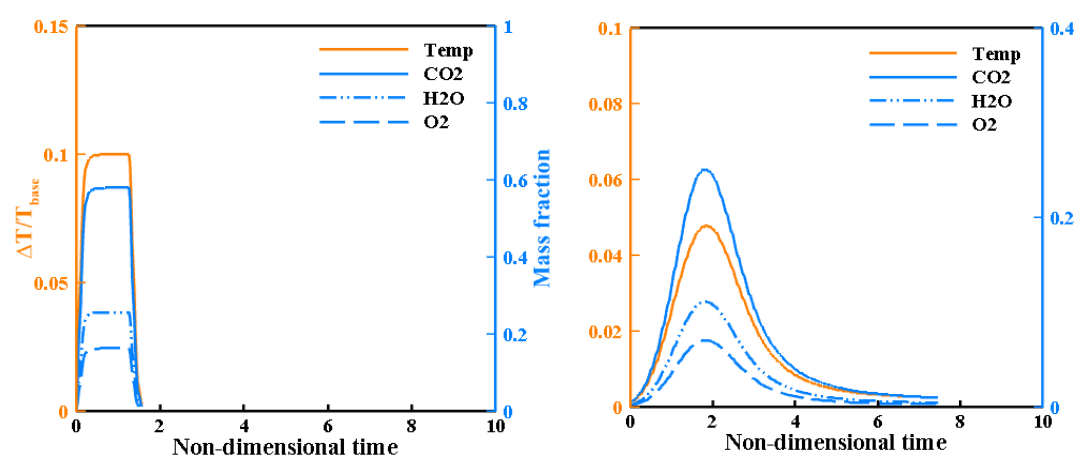

Case 1
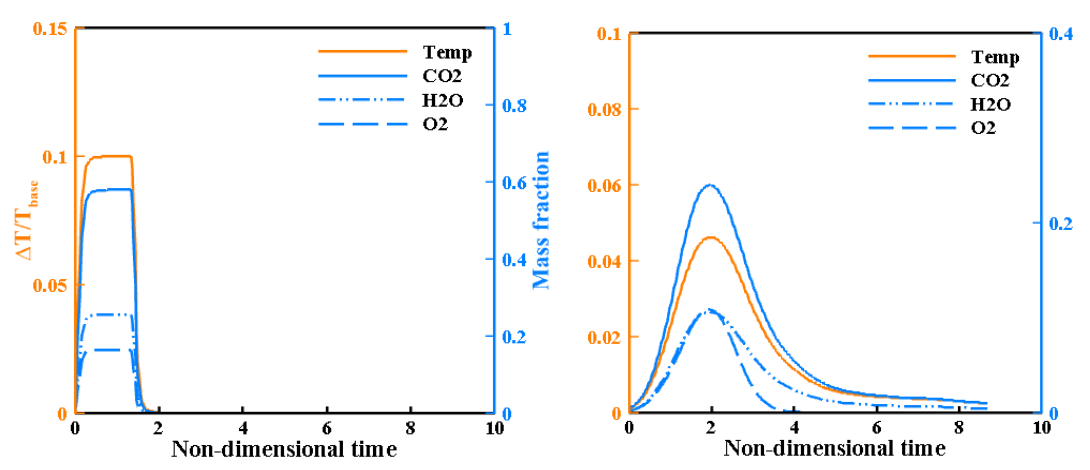

$x / L=0.5$

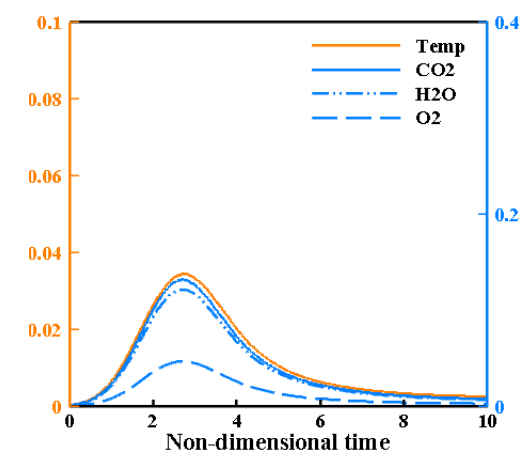

$x / L=0.9$

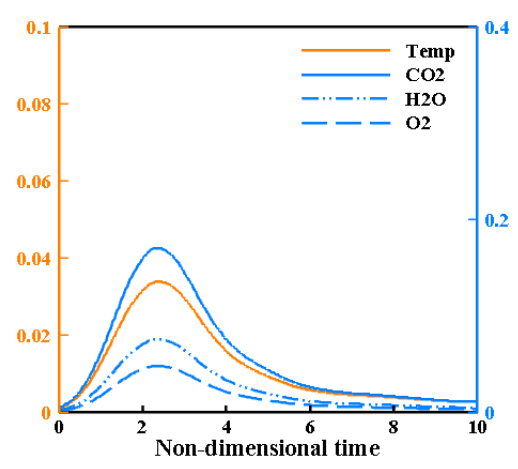

Case 2 

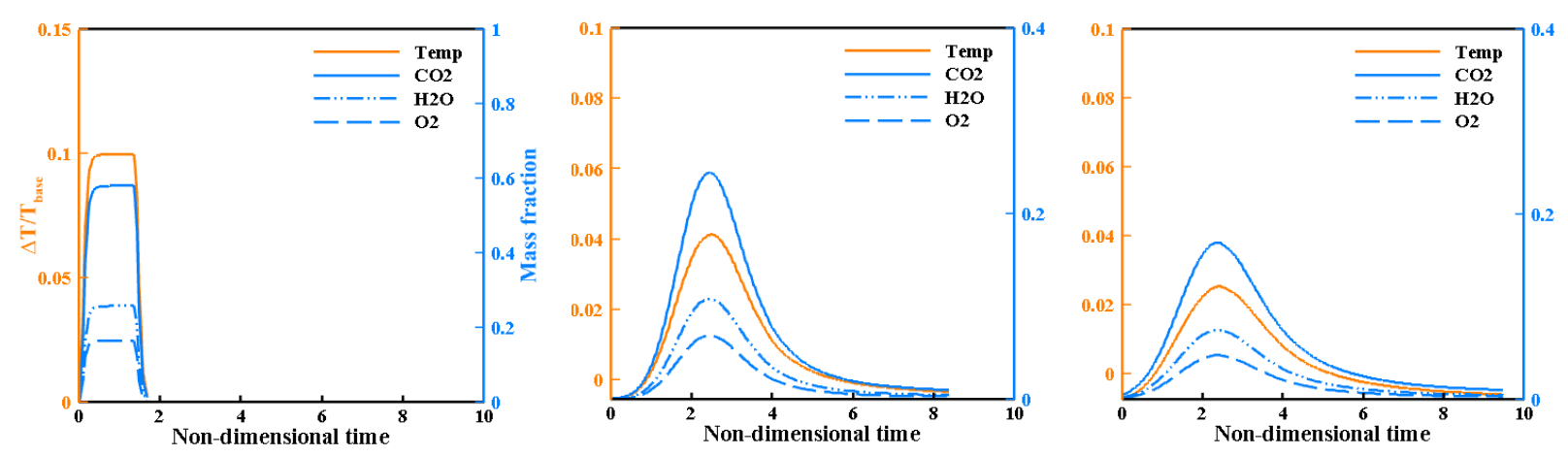

Case 3
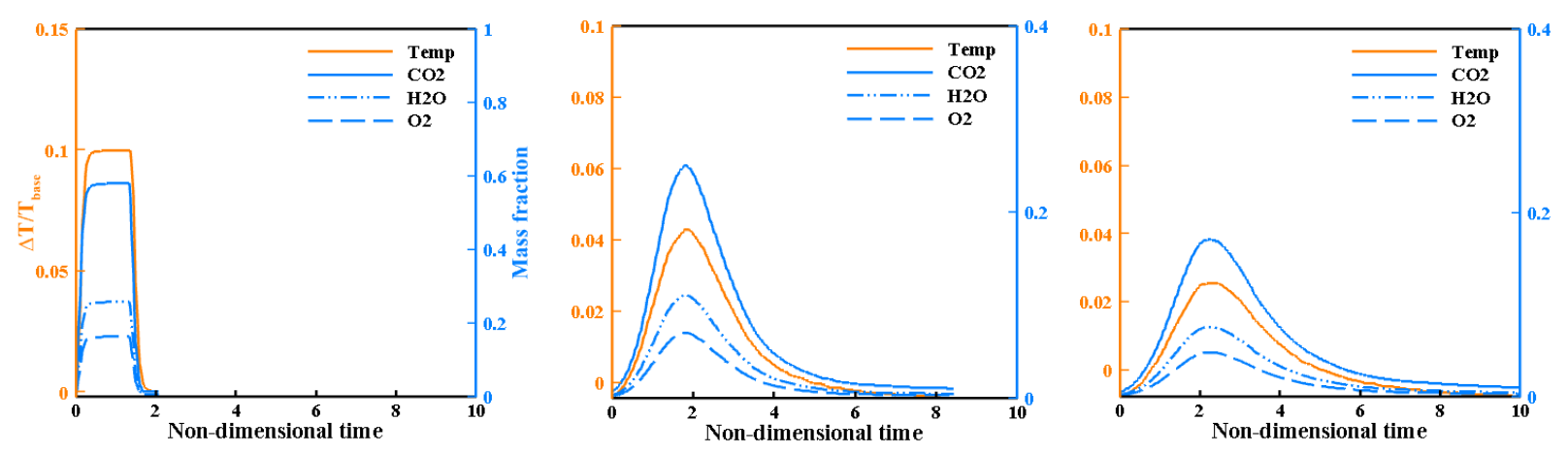

Case 4

Fig. 4. Time-trace of the temperature increment and species concentrations during convection of the wave from the channel inlet to the outlet.

Contours of the sources $S_{1}$ to $S_{3}$ as well as chemical potential and specific Gibbs energy are illustrated in Figs. 5 and 6, respectively for cases 1 and 3. These have been presented for two moments; first, when they have been just produced, and second, when the wave's front touches the location of $x / L=0.9$. The

5 highly coherent waves at the inlet decay significantly to distributed non-uniformities near the outlet. The

6 influence of hydrodynamics is quite clear by dissipating the wave at the near-wall region, as previously

7 shown in the context of entropy waves [20,40]. This degrades the waves by mass and thermal diffusion.

8 The tail of the waves spreads upstream to the inlet. Further, the wave's front is not clearly distinguishable.

9 This is the reason for introducing a yardstick to specify a wave and cut off its unimportant parts in the current study (see Eq. (25)) and earlier investigations [19,20,43]. As shown for case 3 in Fig. 6, further to 
1 the destroying effects of turbulent flow, heat transfer from the channel leads to the wave amplitude to be

2 influenced. This indicates that similar to that in entropy waves, heat transfer can significantly affect the

3 survival of chemical sources of the compositional wave. The evolution of $S_{2}$ is underscored more than the

4 other sources. Due to the cooling of the base fluid flow, the background of chemical potential and $S_{1}$ contour

5 is different to the others in Fig. 6. Clearly, the values of the preceding two terms vary more significantly

6 than others if convective cooling is applied on the walls.

Inlet

$x / L=0.9$
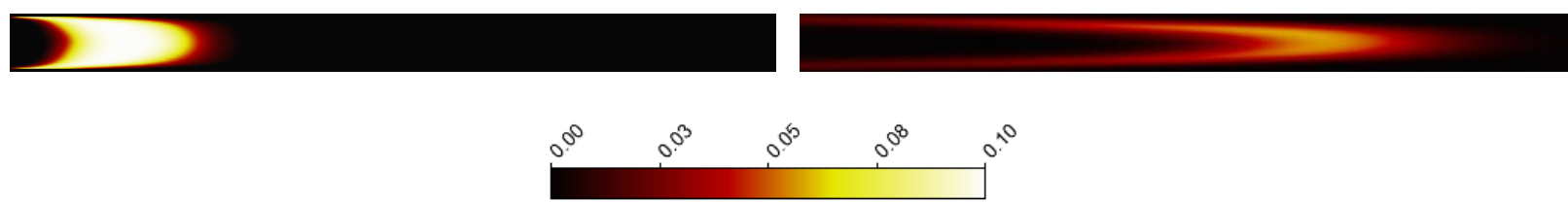

$S_{1}$
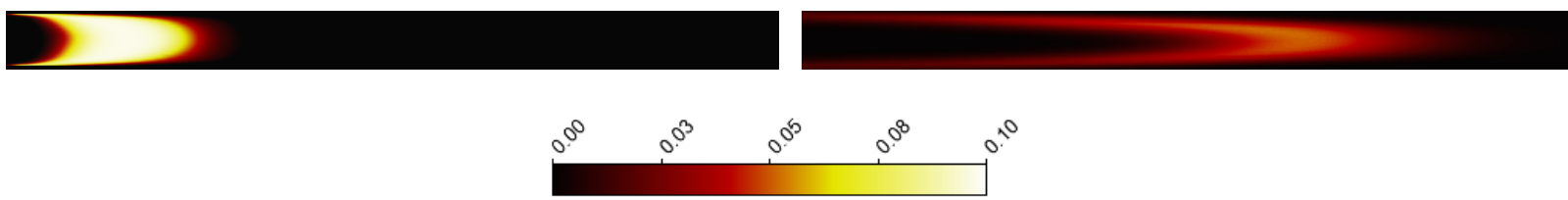

$S_{2}$
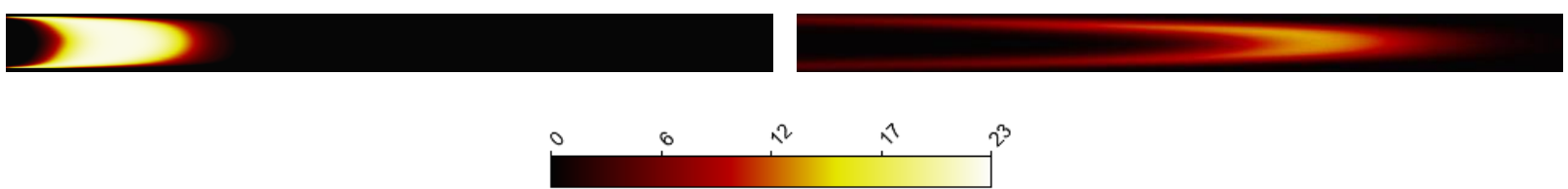

$S_{3}$
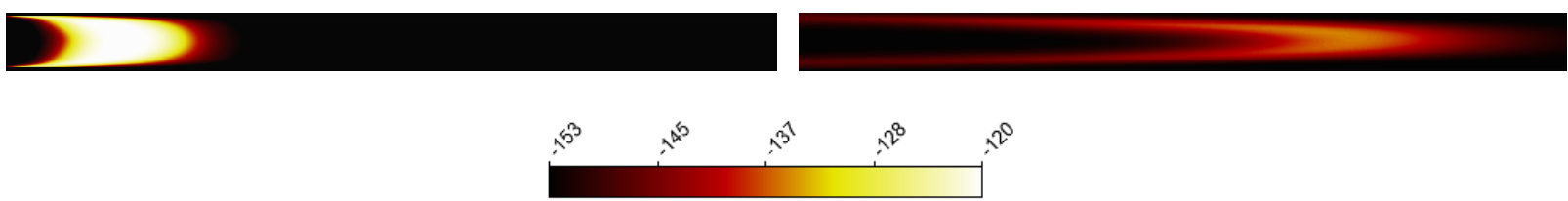

Chemical potential
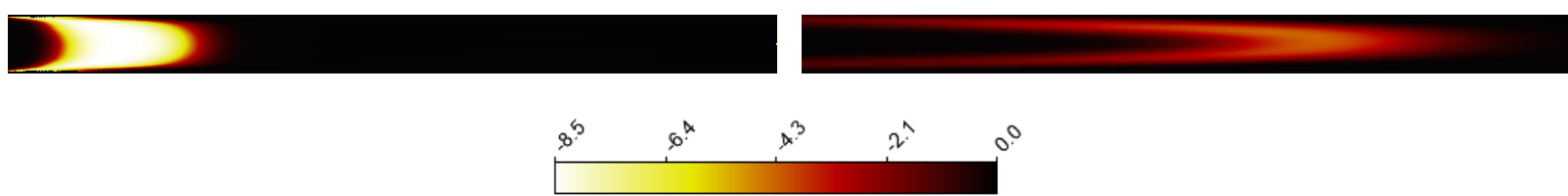

Specific Gibbs energy (MJ/kg) 
Fig. 5. Contours of the sources and other dominant chemical parameters for case 1 at the channel inlet and near the outlet.

Inlet

$x / L=0.9$
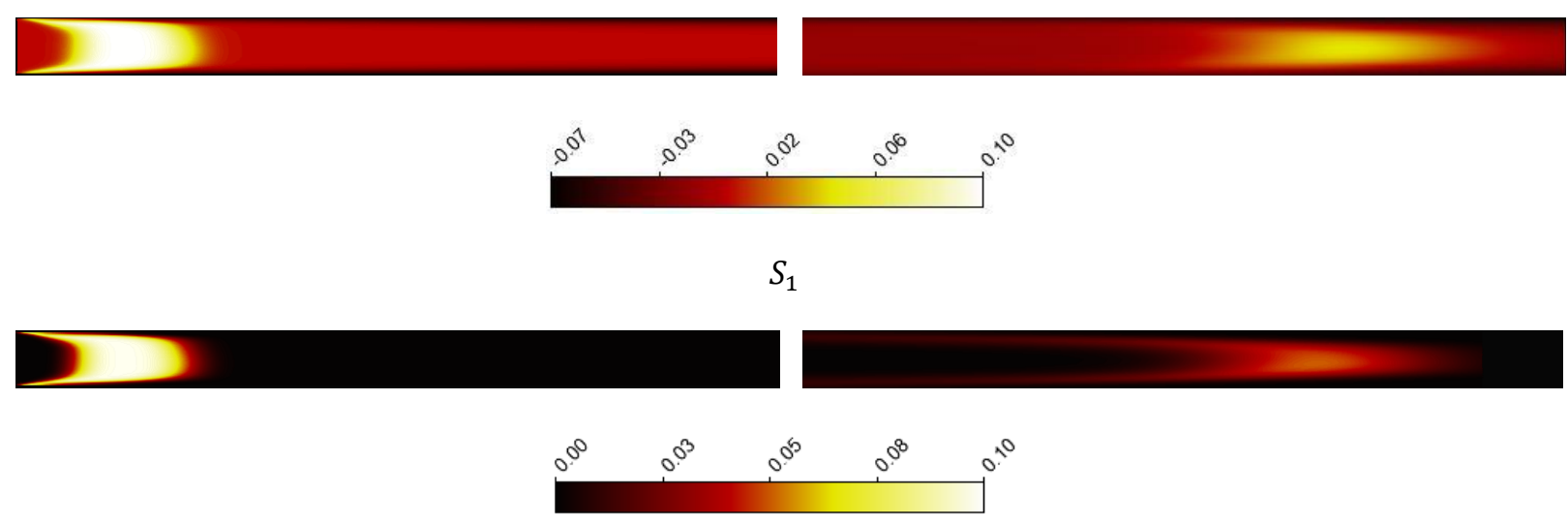

$S_{2}$
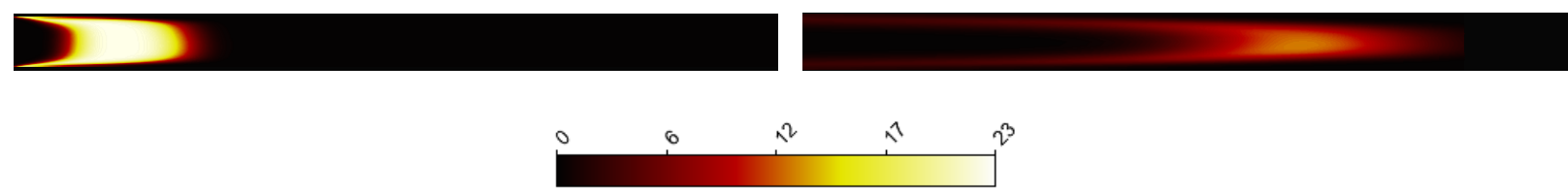

$S_{3}$
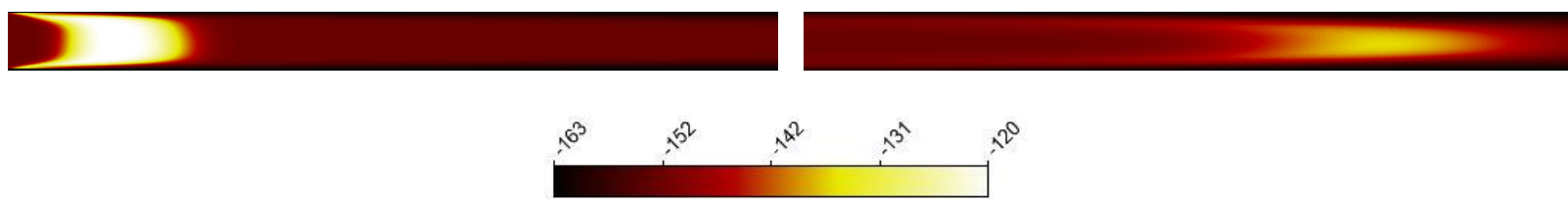

Chemical potential

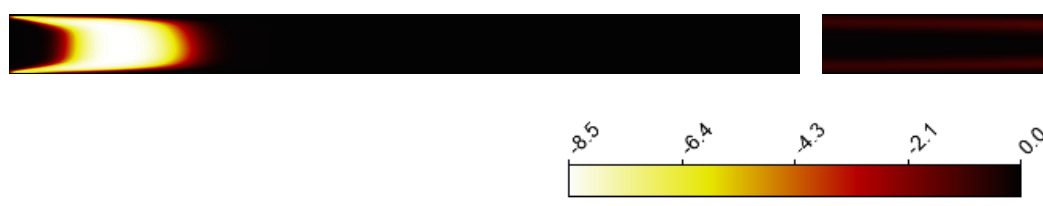

Specific Gibbs energy

Fig. 6. Contours of the sources and other dominant chemical parameters for case 3 at the channel inlet and near the outlet. 
Fig. 7 depicts the variation of coherence function with the wave Strouhal number for the three

2 investigated sources of indirect noise and cases 1 to 4 between inlet and middle as well as inlet and outlet

3 of the channel. The coherence function shows a qualitatively similar pattern for a wave in the middle or the

4 outlet of the channel. However, based on the values of coherence, the wave annihilation is more significant

5 towards the outlet in comparison to the middle of the channel. As a general trend, entropic and

6 compositional waves remain more coherent at low Strouhal numbers, indicating that large-scale features of

7 the wave survive the flow. This has been also reported in the earlier studies of entropy waves $[15,17,24]$.

8 At higher Strouhal numbers, the sources feature a scattered pattern with a low chance of survival and hence

9 noise generation. The described patterns show that chemical sources are influenced similar to the

10 conventional entropic wave. Elevating the turbulence intensity from 5\% to $20 \%$ in the adiabatic case

11 imparts no major effect on the coherence function. It only slightly distorts the wave and the coherence

12 shows more spread. However, this is not the case for the channel with convective heat transfer in which the

13 average value of coherence drops by a factor of 0.66 in the low-frequency range. It is important to note that

14 the current simulations consider air as an ideal gas and therefore the hydrodynamic and thermal fields are

15 coupled. Wall cooling modifies the temperature field and subsequently affects the flow. It appears that heat

16 transfer contribution with wave annihilation is profoundly higher than that of turbulence intensity. This is

17 such that the convective heat losses can significantly scatter the compositional wave at almost all

18 frequencies. The graphs of coherence include local minima at discrete values of Strouhal number. This

19 behavior has been already observed and explained in the previous experimental studies [43,55] and is not

20 further discussed here. Further, there exist strong similarities between the coherence of compositional and

21 entropy waves, implying that their annihilating mechanisms are also similar. Hence, although the physics

22 of the compositional and entropic waves are different, the phenomena that can affect them are the same.

23 These include turbulence, heat transfer, and diffusion.

(a)

(b) (c) 

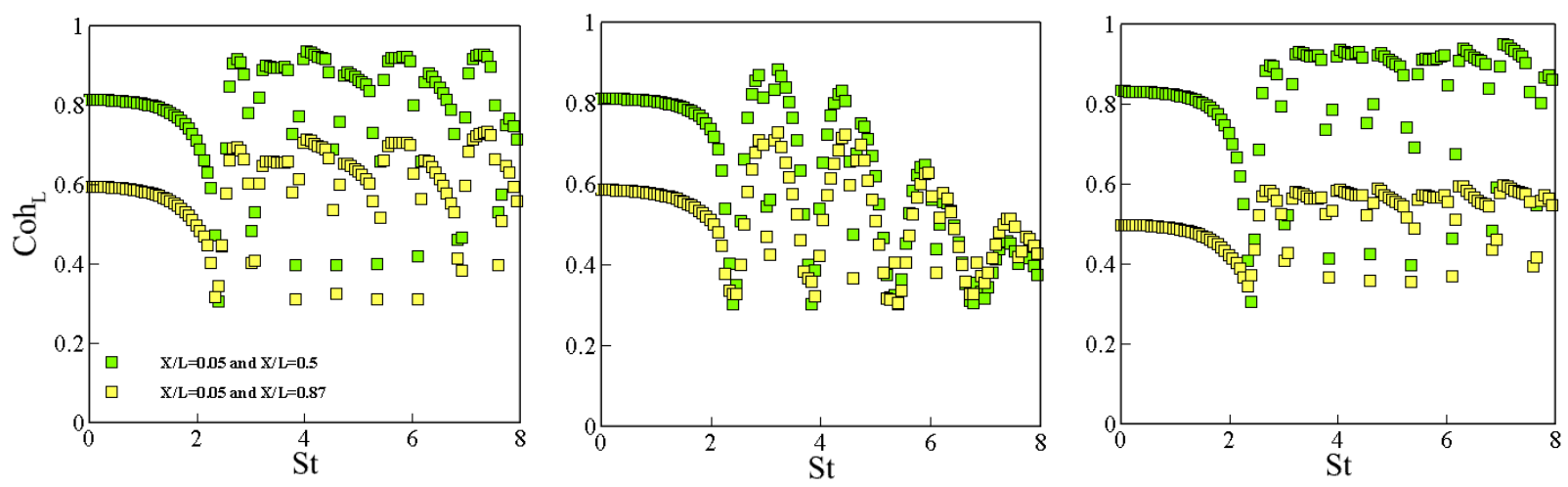

Case 1
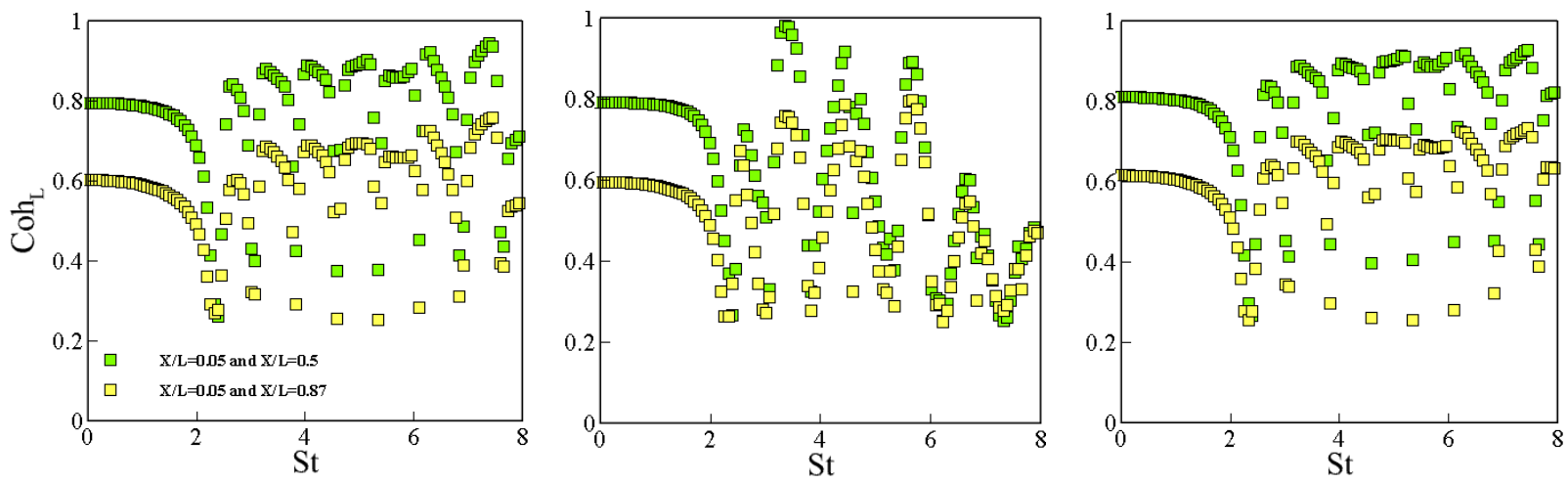

Case 2
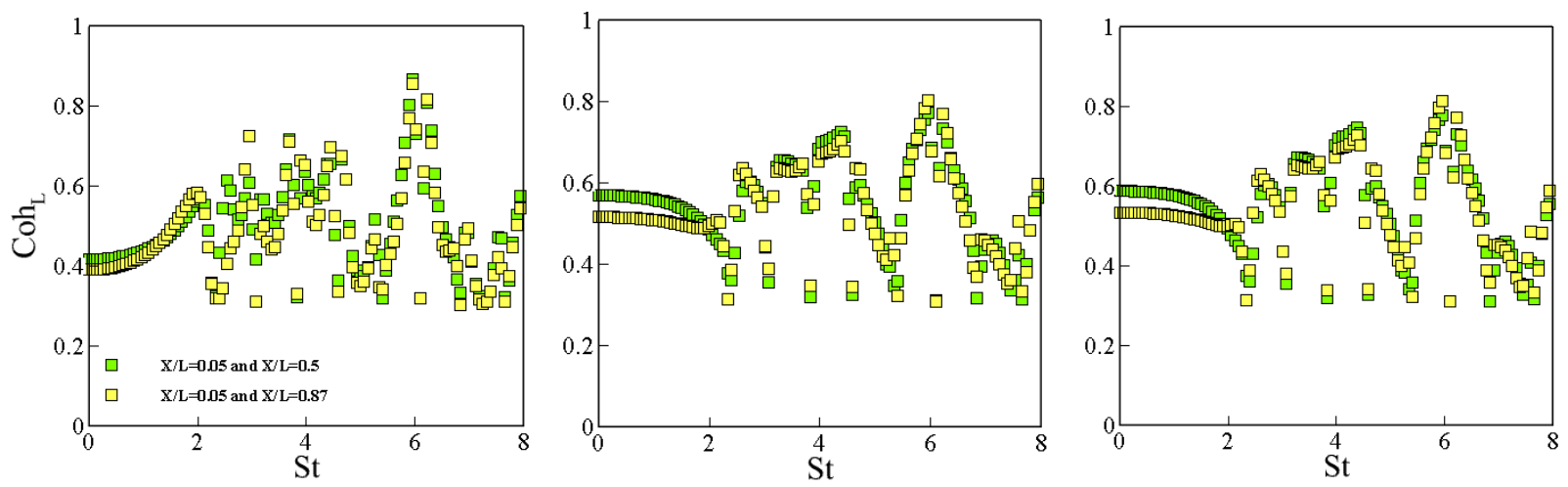

Case 3 

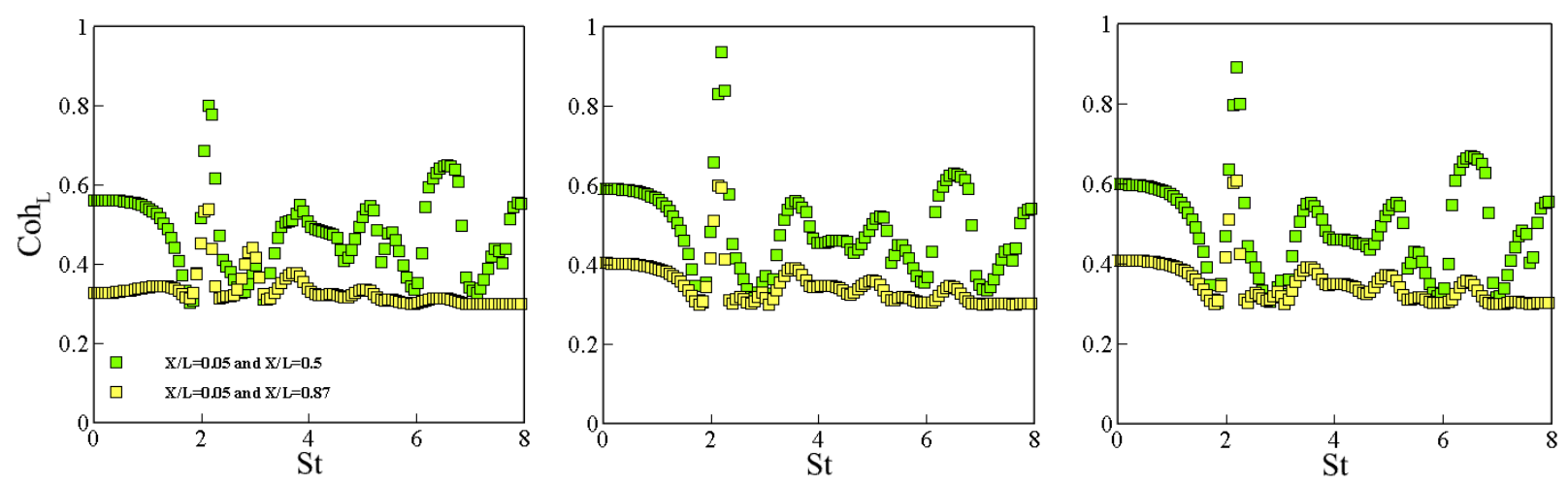

Case 4

Fig. 7. $\mathrm{Coh}_{\mathrm{L}}$ for the sources between the inlet and middle (green dots) as well as the inlet and near the outlet (yellow dots) of the channel for cases 1 to 4 for (a) $s_{1}$, (b) $s_{2}$ and (c) $s_{3}$.

1

The dissipating mechanisms may destroy the wave's front and break it into some distinctive parts. It has been already shown that entropy waves are far from being one-dimensional $[20,43]$. Nonetheless, a corresponding analysis has not been conducted on the compositional waves. To address this issue, the coherence on the wavefront, $\operatorname{Coh}_{D}$, was calculated and the outcomes are presented in Fig. 8. The wavefront is completely correlated at the inlet except for the high-frequency range of the spectrum. In case 4 wherein convective cooling and higher turbulence intensity are applied, the coherence function takes the values lower than unity. As expected, the front coherence drops near the outlet. This is due to the mixing processes that tend to smear out the compositional wave. The patterns are quite similar for the adiabatic cases, with the exception that the coherence values of the troughs are slightly reduced by enhancing the turbulence level. Comparing cases 1 and 2 depicts the effects of turbulence on the wavefront. These appear to be nonnegligible according to the coherence values at the inlet and outlet. As expected, heat transfer on the walls lowers the coherence. The lowest value of the coherence with the most scattered pattern is found for case 4 where it is affected by both turbulence and heat transfer. Fig. 8 implies that for short and adiabatic conduits, the compositional wave is practically one-dimensional. However, the existence of convective heat transfer can easily annihilate the wave and break down the wave one-dimensionality. 
(a)
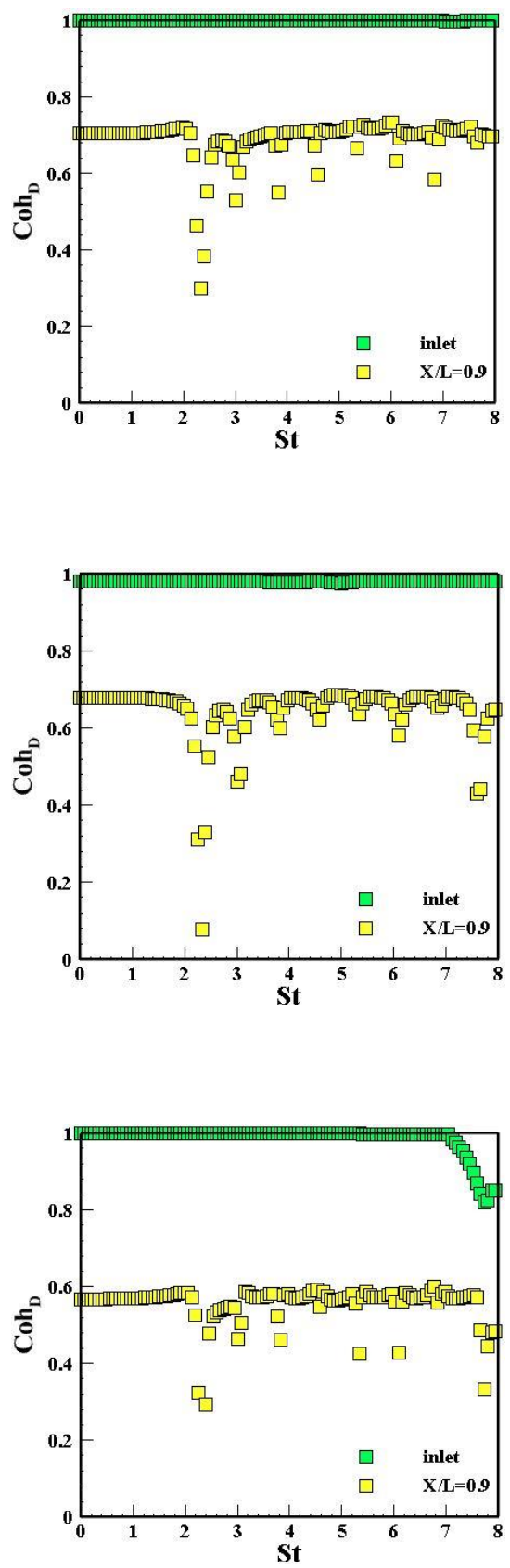

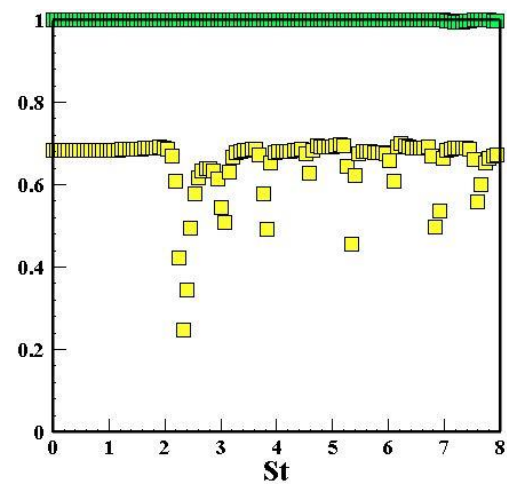

Case 1
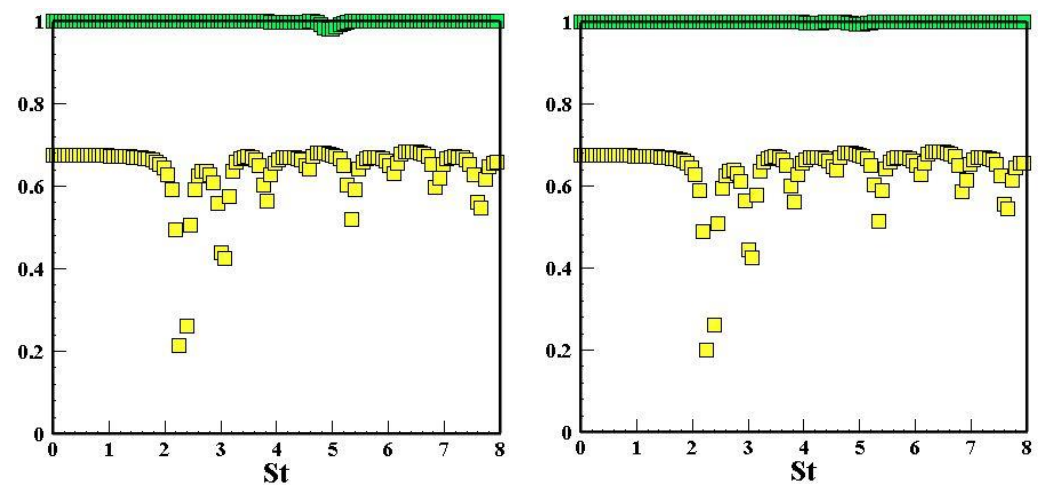

Case 2

(b)

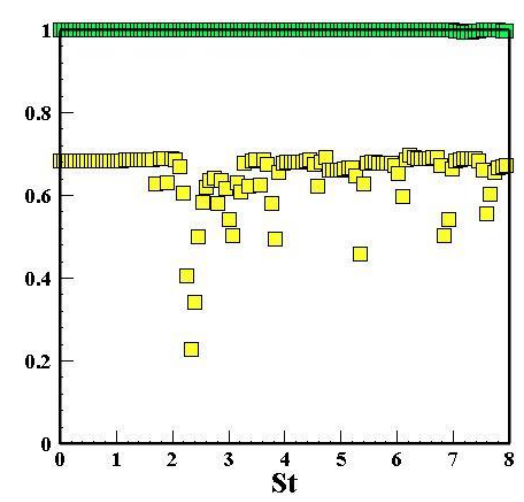

(c)
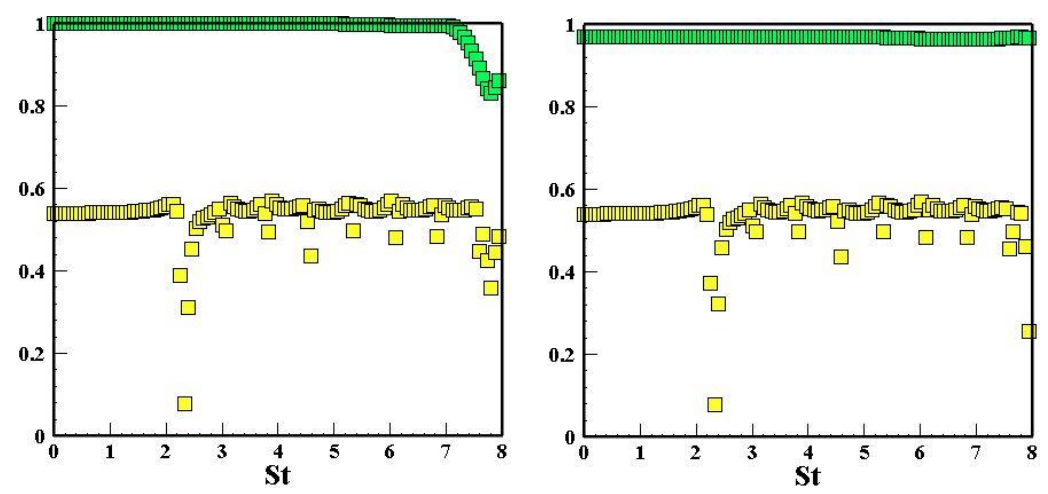

Case 3 

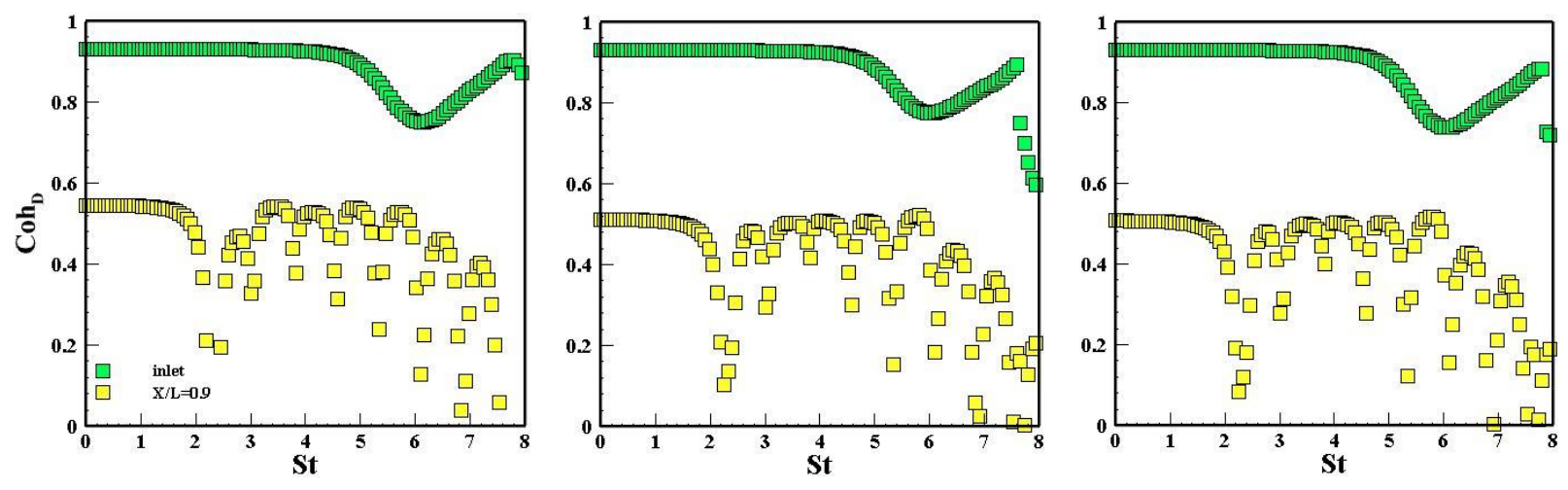

Case 4

Fig. 8. $\mathrm{Coh}_{\mathrm{D}}$ for the sources at the inlet (green dots) and near the outlet (yellow dots) of the channel for cases 1 to

$$
4 \text { for (a) } s_{1} \text {, (b) } s_{2} \text { and (c) } s_{3} \text {. }
$$

Figs. 9 shows the longitudinal coherence of chemical potential function between the inlet and middle as well as the channel inlet and near the outlet during convection of the compositional wave. Similar to that shown earlier, Fig. 9 involves 4 cases with different turbulence intensities and adiabatic and cooled walls. For the adiabatic walls, the coherence function remains close to unity for almost the entire spectrum. Turbulence intensity affects the coherence of chemical potential by producing more and deeper troughs.

7 Convective cooling features qualitatively similar, but stronger effects on the coherence of chemical potential. Nonetheless, regardless of the thermal boundary condition on the walls of the channel or flow

9 turbulence intensity, the coherence of chemical potential is generally high and mostly close to unity. Fig. 10 involves similar information to Fig. 9, except for the front of the wave. For high frequencies, turbulence

11 intensity increment and convective cooling have a minor effect on the front coherence at the inlet. Heat

12 transfer on the walls, however, appears to be more influential. In the vicinity of the outlet, the coherence

13 limitedly falls at some discrete Strouhal numbers, while it still remains unaffected. The behavior depicted 14 in the last two figures is in sharp contrast with that observed in parts $\mathrm{b}$ and $\mathrm{c}$ of Fig. 7 showing the coherence 15 of $s_{3}$ and featuring a significant drop especially at higher frequencies. Therefore, the changes in $s_{3}$ 16 coherence are dominated by the mass fraction of species and mixture fraction and not by the chemical 
1 potential function. During convection of the compositional wave, the mixture fraction and mass fraction of 2 species are both expected subject to a strong decay.

(a)
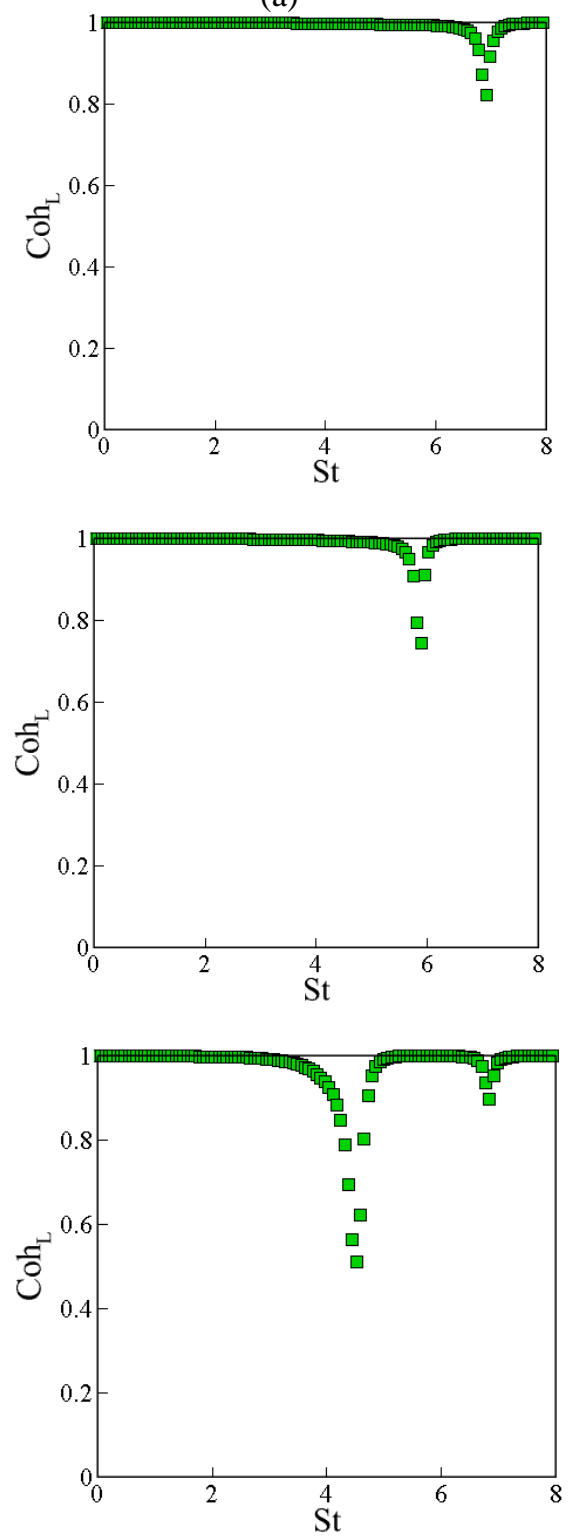

(b)

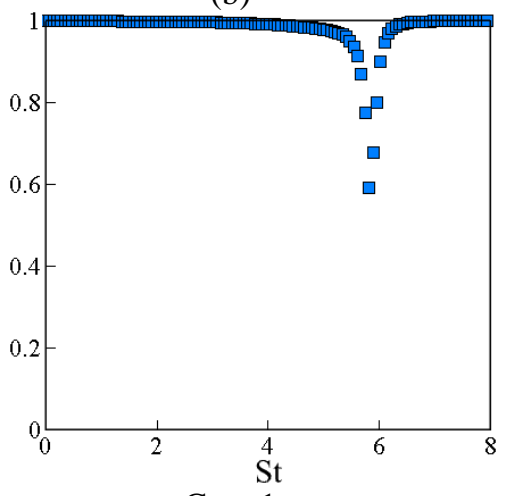

Case 1

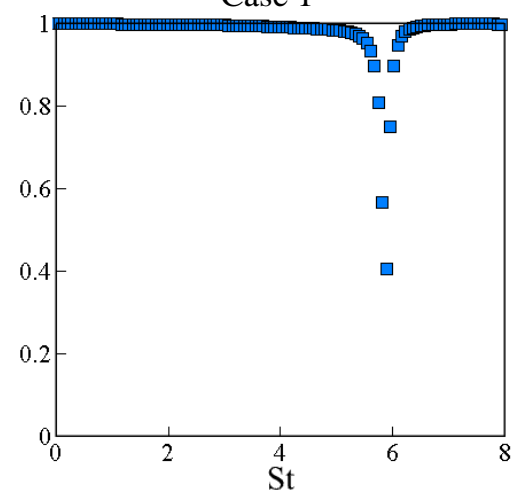

Case 2

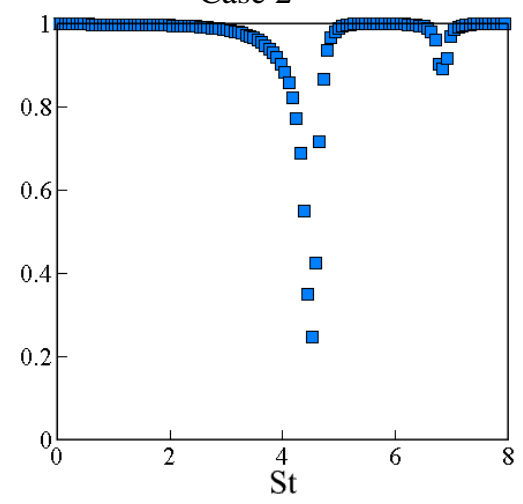

Case 3 (c)
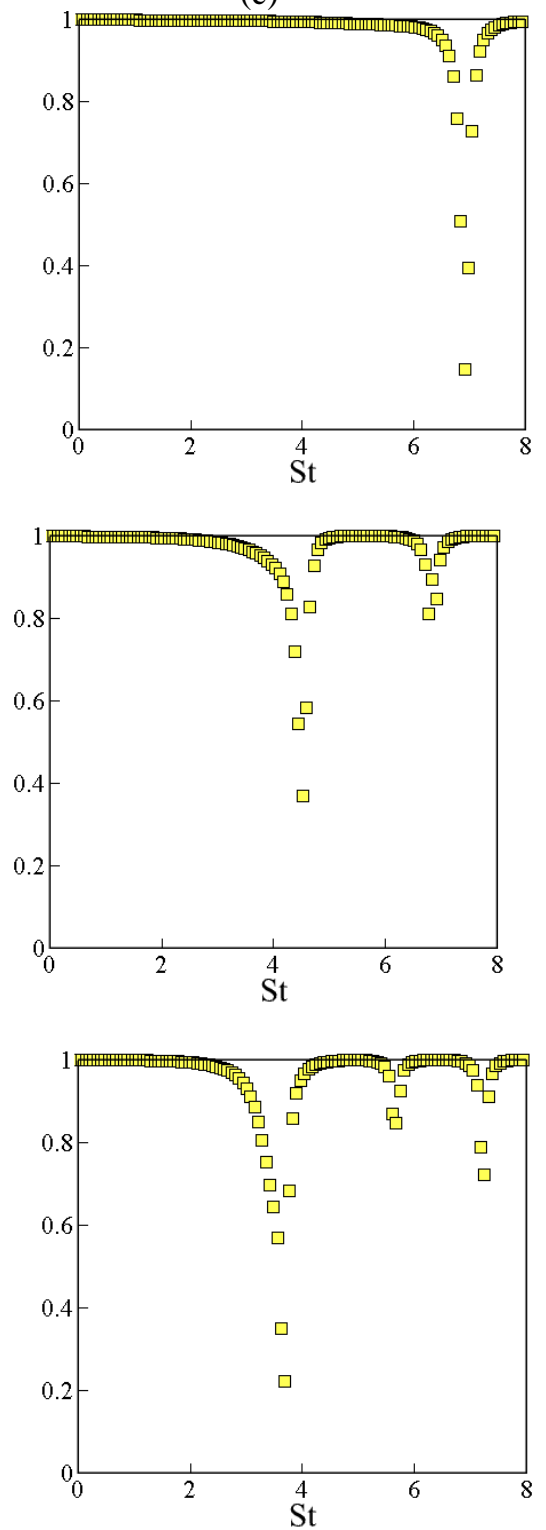

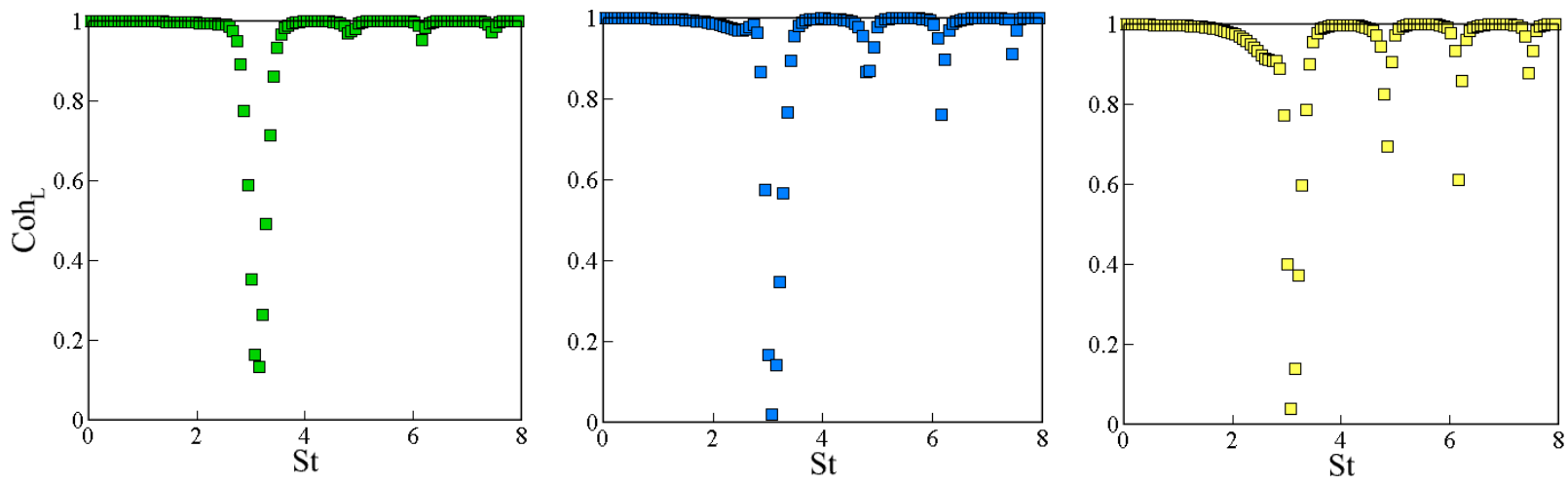

Case 4

Fig. 9. $\mathrm{Coh}_{\mathrm{L}}$ for chemical potential function $(\Psi)$ for cases 1 to 4 between the inlet and (a) $x / L=0.25$, (b) $x / L=0.5$ and (c) $x / L=0.9$.

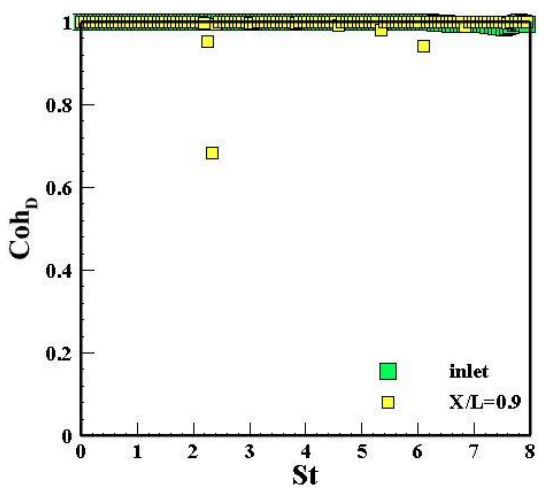

Case 1

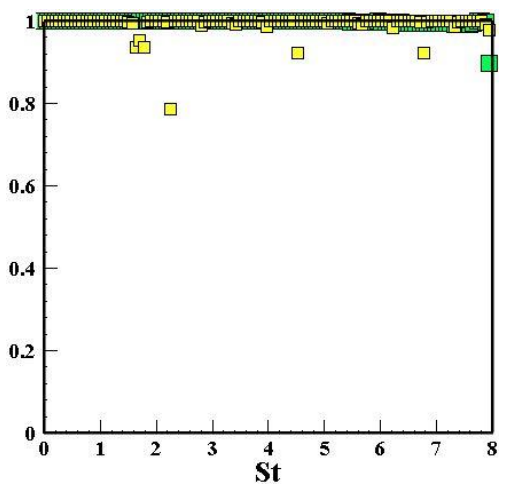

Case 3

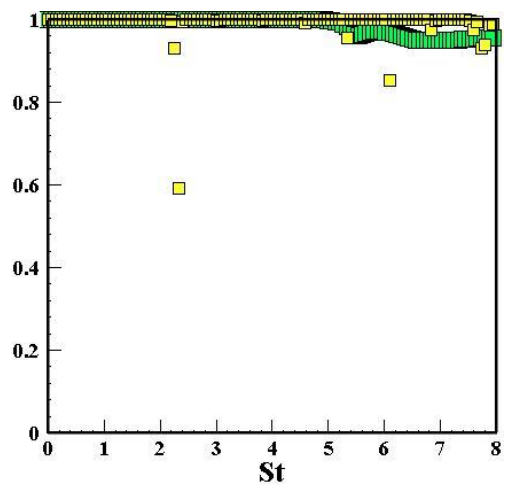

Case 2

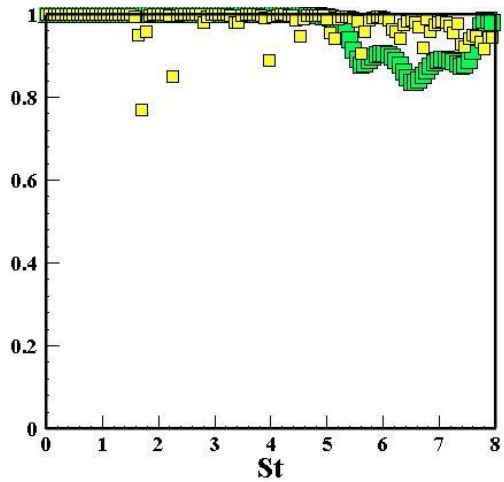

Case 4

Fig. 10. $\mathrm{Coh}_{D}$ for chemical potential function $(\Psi)$ for cases 1 to 4 for the inlet and near the outlet location.

Eq. (16) implies that temperature and chemical potential can be spatially related. Their relation

4 through frequency domain is examined in Fig. 11, which is set in the similar way as the previous figures. 
1 This figure draws an analogy amongst the factors that make a decay in temperature and chemical potential.

2 For the Strouhal numbers lower than 2, the coherence is fixed at almost unity. As the Strouhal number

3 increases, the relation starts to vary. This is more pronounced for the cases with convective heat transfer or

4 higher turbulence intensity; however, the effect of the latter is more significant than that of the former. The

5 poorest relation is found in case 4 where heat transfer exists and turbulence intensity further contributes to

6 wave annihilation. The coherence falls in value as the wave continues to pass through the duct. This is,

7 however, not the case for the low Strouhal number $(S t<2)$ components of the wave. It follows that for the

8 low-frequency components, attenuation of the temperature gradient can weaken the chemical potential.

9 Thus, both sources of 1 and 3 can be affected by the hydrodynamic or thermal mechanisms, owing to the

10 degradation of the temperature gradient. At higher Strouhal numbers, the relation between chemical

11 potential and temperature is less significant, indicating that the influence of decaying mechanisms on them

12 has varied. This figure indicates that albeit $s_{3}$ is a chemical source, it is not solely related to the chemical

13 phenomena in the post flame zone of the combustor and can be altered by the flow cooling, commonly

14 occurring in gas turbine combustors.

(a)

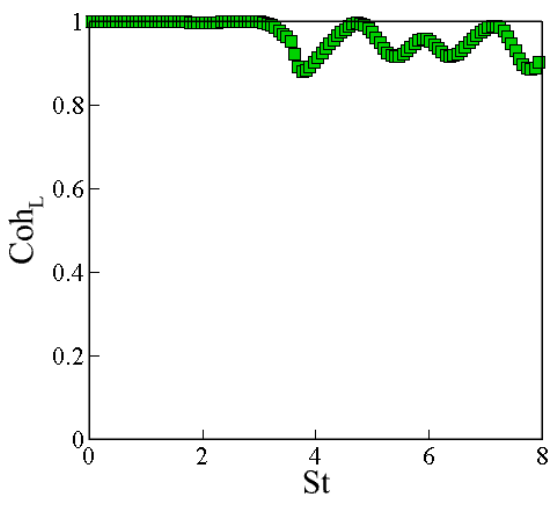

(b)

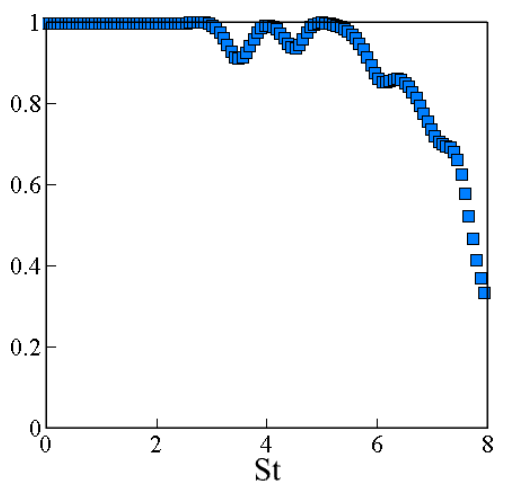

Case 1 (c)

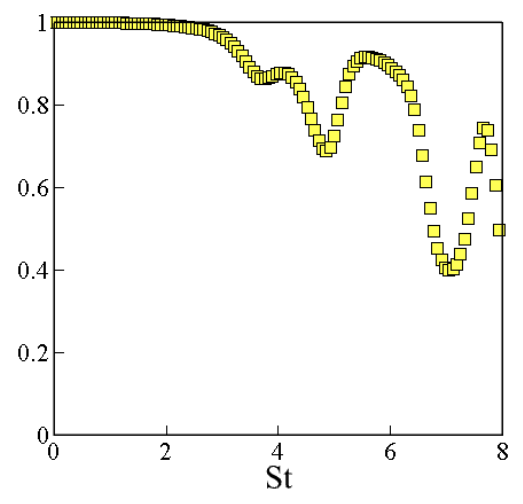



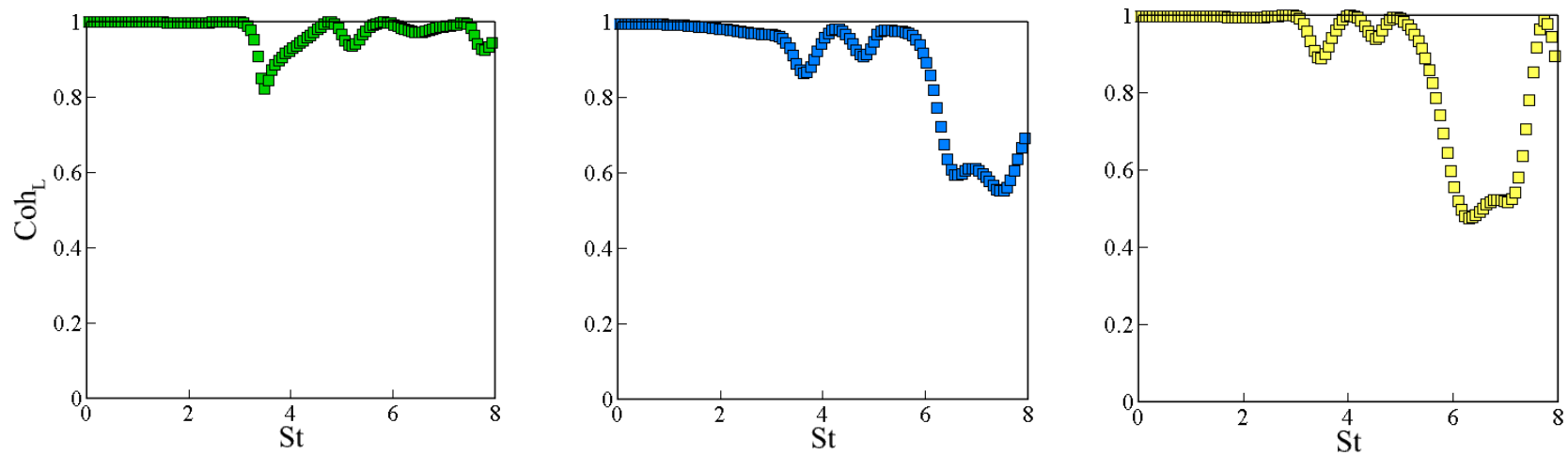

Case 2
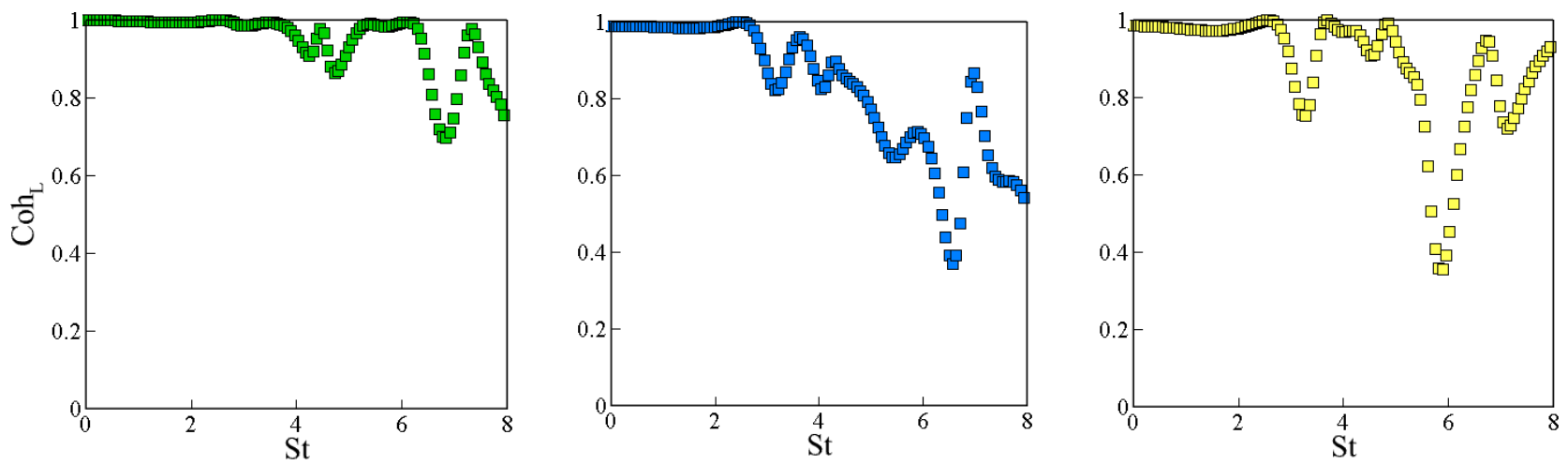

Case 3
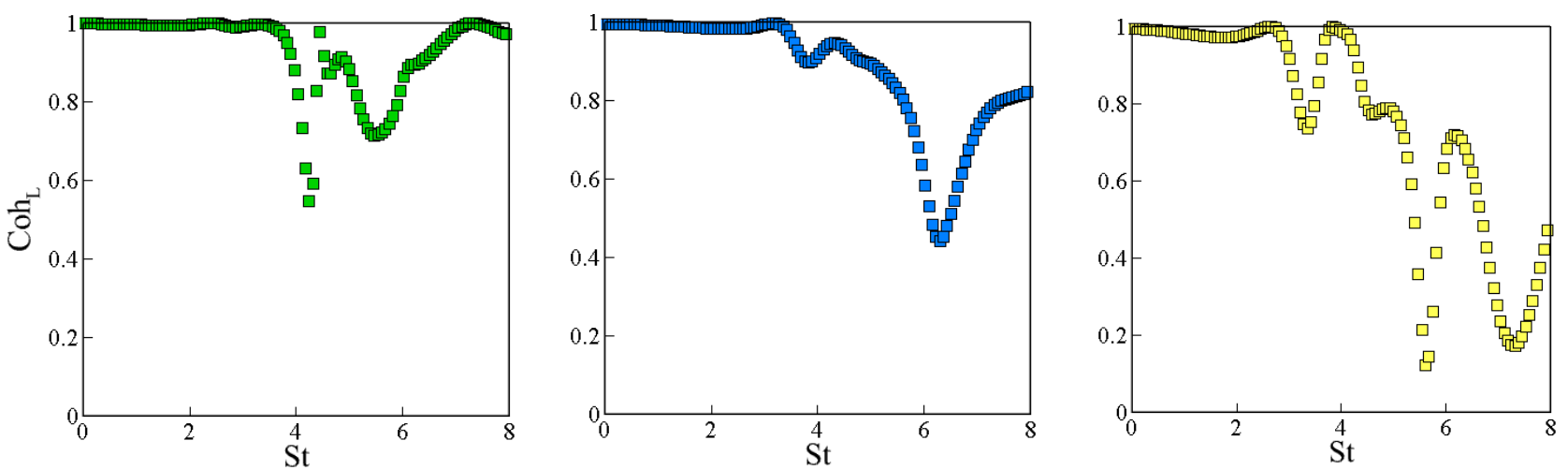

Case 4

Fig. 11. The coherence for chemical potential function $(\Psi)$ and temperature for case 1 to 4 (a) $x / L=0.05,($ b) $x / L=0.5$ and (c) $x / L=0.9$.

The dissipation index for the three investigated sources is demonstrated in Fig. 12. These have been

3 calculated using the mass-averaged of the sources' amplitude (see Eq. (26)). In keeping with the earlier 4 current results and previous investigations [20,33,57], this figure shows that the entropic wave is more 
1 strongly annihilated at higher frequencies. It also reveals that the compositional sources are highly affected

2 by the flow thermo-hydrodynamics at high frequencies. For the first time, the current results show that a

3 compositional source is dissipated qualitatively similar to the entropic wave, but more severely. This stems

4 from the nature of the compositional sources affected by both thermal and chemical factors. The dissipation

5 at high frequencies and near the outlet dramatically upraises to $80 \%$ for the adiabatic cases and reaches

$6 \quad 100 \%$ for the heat transferring cases. However, the dissipation at the low frequencies can be about fourfold

7 lower than those of high frequencies. On average, the compositional sources involve about $10 \%$ and $20 \%$

8 more dissipation than the entropic source for the adiabatic and heat transferring cases, respectively.

9 Therefore, it can be concluded that compared to entropy waves, compositional waves are more prone to

10 dissipating mechanisms. Although the noise level generated by different sources is directly related to the

11 subcritical or supercritical nozzle's regime [51], the compositional waves are less likely to survive and thus

12 generate noise. Figure 12 shows that entopic waves are more annihilated in the first half of the channel in

13 comparison to that in the second half. As the Strouhal number increases, the difference between the

14 dissipation of the two halves approaches zero. On the other hand, the compositional waves show a somehow

15 similar degeneration in both halves. It is then concluded that the length of the combustor plays a central

16 role in decaying the compositional wave, as similarly demonstrated for entropy waves $[19,20,43]$. However,

17 this is more underscored for compositional sources. Turbulence intensity increment makes higher

18 dissipation, but not as much as those caused by the convective heat transfer. While the former can intensify

19 the dissipation by $5-10 \%$, the latter increases it by a factor of 2 and limits its survival to very low

20 frequencies. The highest level of dissipation is found in case 4 for which turbulence intensity is high in a

21 heat transferring mean flow.

(a)

(b) (c) 

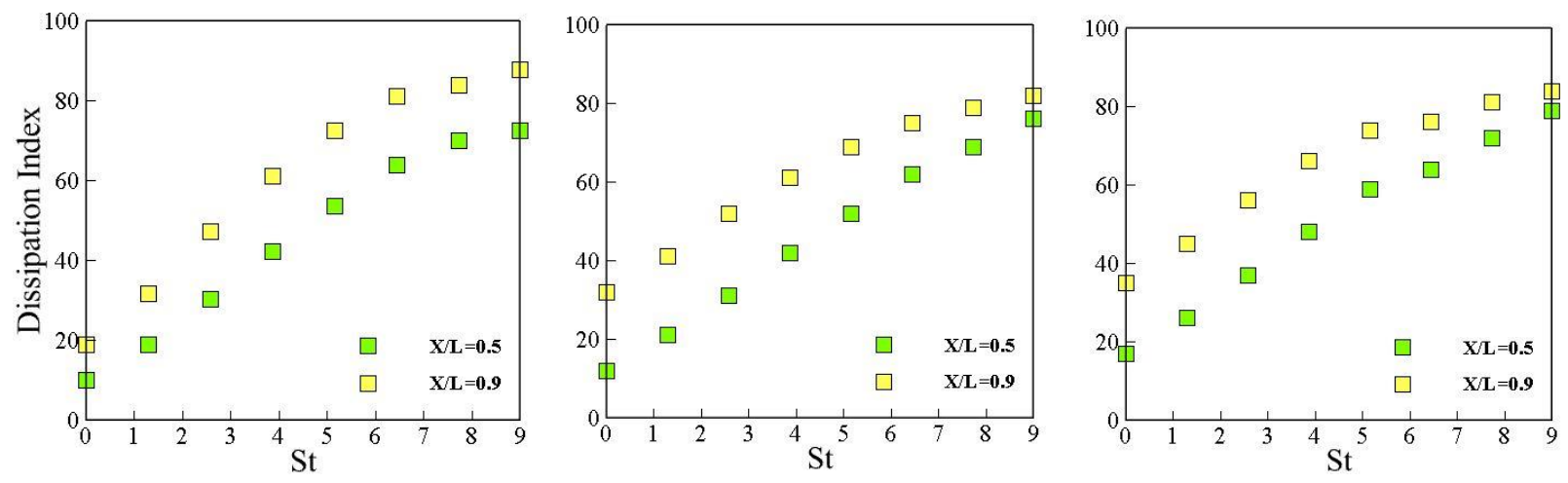

Case 1
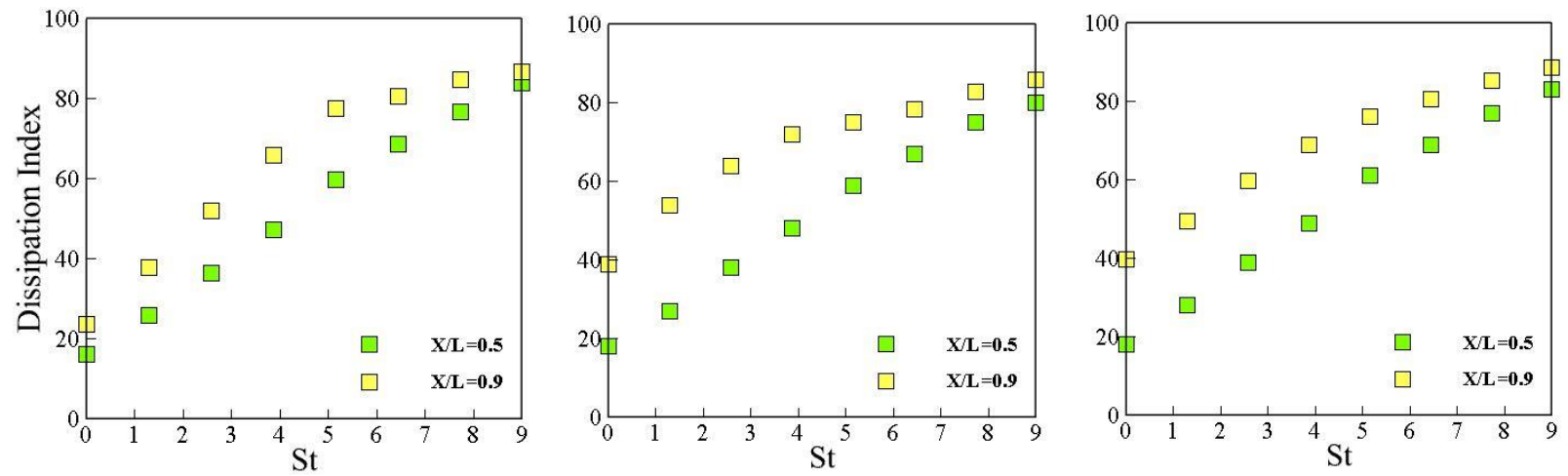

Case 2
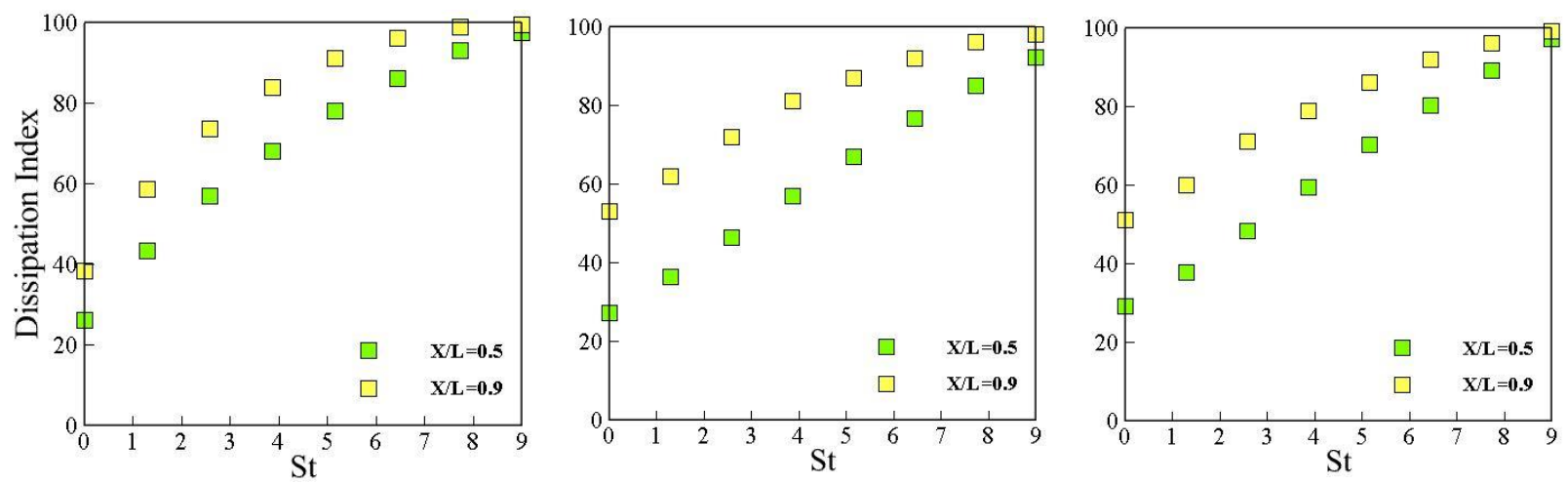

Case 3 

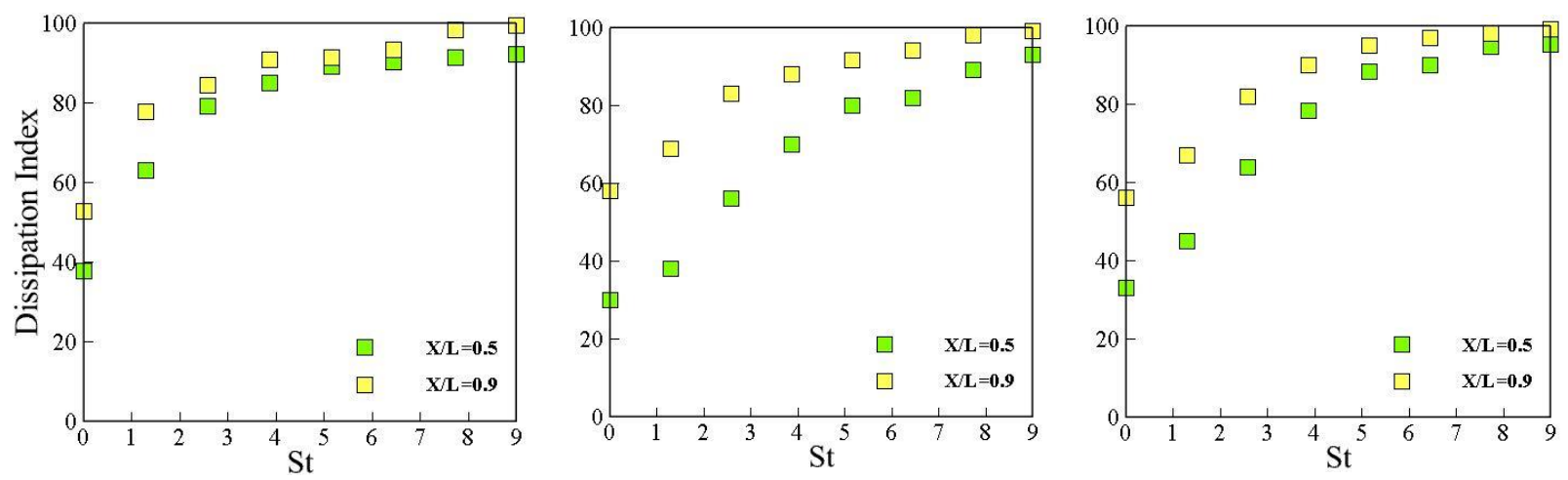

Case 4

Fig. 12. The dissipation index for cases investigated and sources of (a) $s_{1}$, (b) $s_{2}$ and (c) $s_{3}$.

\section{Conclusions}

3 Decay of the sources of indirect combustion noise is of significance as it can partially or even completely

4 suppress noise generation. For this reason, the annihilation of entropy waves has been investigated in the

5 recent past. However, so far, there has been no study on the deterioration of compositional waves as the

6 recently introduced source of indirect combustion noise. The current study, therefore, shed some light on

7 the fate of convecting compositional waves. This was done through a large eddy simulation of a channel

8 flow with a temporal pulse of hot combustion products at the inlet, which was then convected by the mean

9 flow. The effects of turbulence level of the mean flow and thermal boundary conditions of the channel, i.e.

10 adiabatic and convective cooling, were evaluated. A statistical assessment was performed in the frequency

11 domain to find out how a compositional wave might decay. Further, the annihilation of compositional

12 sources of indirect combustion noise was compared with that of the thermal source. The key findings of

13 this study can be summarized in the followings.

- Compared to turbulence intensity of the mean flow, convective heat transfer in the investigated cases made a more significant contribution. 
- The mixture fraction variation was chiefly responsible for the compositional wave's decay, whereas the contribution of chemical potential was comparatively minor. Therefore, mixture fraction variation was considered as the sink of the compositional wave.

- Due to the strong correlation between the chemical potential and temperature disturbance at low frequencies, any annihilating mechanism that could decay one could do the same to the other.

- Compared to the thermal source, the compositional sources were more strongly affected by the flow field; it elicited up to $20 \%$ higher values in the dissipation index and therefore lower contribution of the compositional waves with the indirect noise generation. This stems from the nature of the compositional sources affected by both thermal and chemical factors.

- The dissipation of compositional wave at low frequencies could be about four times lower than those at high frequencies.

- Similar to that for entropy waves, the channel length plays an essential role in the survival of compositional waves.

\section{Acknowledgment}

N. Karimi acknowledges the financial support of EPSRC through the grant number EP/V036777/1. A. Fattahi would like to thank the University of Kashan to support this work under Grant No. 1073239.

\section{References}

[1] Morgans AS, Duran I. Entropy noise: A review of theory, progress and challenges. JournalsSagepubCom 2016;8:285-98. https://doi.org/10.1177/1756827716651791.

[2] Lieuwen T, Lieuwen T. Modeling Premixed CombustionnAcoustic Wave Interactions: A Review Non-linear Flame Response Physics View project Blowout of swirl dump combustors View project Modeling Premixed Combustion-Acoustic Wave Interactions: A Review. Artic J Propuls Power 2003;19:765-81. https://doi.org/10.2514/2.6193. 
1 [3] Leyko M, Nicoud F, Poinsot T. Comparison of direct and indirect combustion noise mechanisms in a model combustor. AIAA J 2009;47:2709-16. https://doi.org/10.2514/1.43729.

3 [4] Strahle WC. Combustion noise. Prog Energy Combust Sci 1978;4:157-76.

4 [5] Dowling AP, Mahmoudi Y. Combustion noise. Proc Combust Inst 2015;35:65-100.

5 [6] Chiu HH, Summerfield M. Theory of combustion noise. Acta Astronaut 1974;1:967-84.

6 [7] Chu B-T, Kovásznay LSG. Non-linear interactions in a viscous heat-conducting compressible gas.

$7 \quad$ J Fluid Mech 1958;3:494-514.

8 [8] Strahle WC. Duality, dilatation, diffusion and dissipation in reacting turbulent flows. Symp.

$9 \quad$ Combust., vol. 19, Elsevier; 1982, p. 337-47.

10 [9] Mahmoudi Y, Dowling AP, Stow SR. Acoustic and Entropy Waves in Nozzles in Combustion $11 \quad$ Noise Framework. Https://DoiOrg/102514/1J055597 2017;55:2369-81.

12 https://doi.org/10.2514/1.J055597.

13 [10] Strahle WC, Muthukrishnan M. Correlation of combustor rig sound power data and theoretical basis of results. AIAA J 1980;18:269-74. https://doi.org/10.2514/3.50759.

[11] Williams JEF, Howe MS. The generation of sound by density inhomogeneities in low MacH number nozzle flows. J Fluid Mech 1975;70:605-22. https://doi.org/10.1017/S0022112075002224.

[12] Marble FE, Candel SM. Acoustic disturbance from gas non-uniformities convected through a nozzle. J Sound Vib 1977;55:225-43. https://doi.org/10.1016/0022-460X(77)90596-X.

[13] Hosseinalipour SM, Fattahi A, Karimi N. Investigation of the transmitted noise of a combustor exit nozzle caused by burned hydrogen-hydrocarbon gases. Int J Hydrogen Energy 2016;41:207586. https://doi.org/10.1016/j.ijhydene.2015.10.119. 
1 [14] Hosseinalipour SM, Fattahi A, Karimi N. Analytical investigation of non-adiabatic effects on the dynamics of sound reflection and transmission in a combustor. Appl Therm Eng 2016;98:553-67. https://doi.org/10.1016/j.applthermaleng.2015.12.116.

[15] Karimi N, Brear MJ, Moase WH. Acoustic and disturbance energy analysis of a flow with heat communication. J Fluid Mech 2008;597:67.

[16] Goh CS, Morgans AS. Phase prediction of the response of choked nozzles to entropy and acoustic disturbances. J Sound Vib 2011;330:5184-98.

[17] Leyko M, Moreau S, Nicoud F, Poinsot T. Numerical and analytical modelling of entropy noise in a supersonic nozzle with a shock. J. Sound Vib., vol. 330, Academic Press; 2011, p. 3944-58. https://doi.org/10.1016/j.jsv.2011.01.025.

[18] Ullrich WC, Bake F, Kings N, Sattelmayer T. Numerical investigation of indirect noise generation by accelerated vorticity. 21st AIAA/CEAS aeroacoustics Conf., 2015, p. 2382.

[19] Hosseinalipour SM, Fattahi A, Afshari H, Karimi N. On the effects of convecting entropy waves on the combustor hydrodynamics. Appl Therm Eng 2017;110:901-9.

[20] Fattahi A, Hosseinalipour SM, Karimi N. On the dissipation and dispersion of entropy waves in heat transferring channel flows. Phys Fluids 2017;29:87104.

[21] Bake F, Michel U, Roehle I. Investigation of entropy noise in aero-engine combustors. J Eng Gas Turbines Power 2007;129:370-6. https://doi.org/10.1115/1.2364193.

[22] Rolland EO, De Domenico F, Hochgreb S. Direct and indirect noise generated by entropic and compositional inhomogeneities. J Eng Gas Turbines Power 2018;140.

[23] Bake F, Kings N, Roehle I. Fundamental mechanism of entropy noise in aero-engines: Experimental investigation. J Eng Gas Turbines Power 2008;130. 
[24] Bake F, Richter C, Mühlbauer B, Kings N, Röhle I, Thiele F, et al. The Entropy Wave Generator (EWG): A reference case on entropy noise. J Sound Vib 2009;326:574-98. https://doi.org/10.1016/j.jsv.2009.05.018.

[25] Sattelmayer T. Influence of the combustor aerodynamics on combustion instabilities from equivalence ratio fluctuations. J Eng Gas Turbines Power 2003;125:11-9.

[26] Eckstein J, Sattelmayer T. Low-order modeling of low-frequency combustion instabilities in aeroengines. J Propuls Power 2006;22:425-32. https://doi.org/10.2514/1.15757.

[27] Eckstein J, Freitag E, Hirsch C, Sattelmayer T. Experimental study on the role of entropy waves in low-frequency oscillations in a RQL combustor. J Eng Gas Turbines Power 2006;128:264-70. https://doi.org/10.1115/1.2132379.

[28] Brear MJ, Carolan DC, Karimi N. Dynamic response of the exit nozzle of a premixed combustor to pressure and entropic disturbances. Proc. 8th Euromech Fluid Mech. Conf. Bad Reichenhall, Ger., 2010.

[29] Hield PA, Brear MJ. Comparison of open and choked premixed combustor exits during thermoacoustic limit cycle. AIAA J., vol. 46, 2008, p. 517-26. https://doi.org/10.2514/1.32650.

[30] Duran I, Moreau S. Solution of the quasi-one-dimensional linearized Euler equations using flow invariants and the Magnus expansion. J Fluid Mech 2013;723:190-231. https://doi.org/10.1017/jfm.2013.118.

[31] Goh CS, Morgans AS. The influence of entropy waves on the thermoacoustic stability of a model combustor. Combust Sci Technol 2013;185:249-68. https://doi.org/10.1080/00102202.2012.715828.

[32] Goh CS, Morgans AS. The influence of entropy waves on the thermoacoustic stability of a model combustor. Combust Sci Technol 2013;185:249-68. 
https://doi.org/10.1080/00102202.2012.715828.

[33] Hosseinalipour SM, Fattahi A, Khalili H, Tootoonchian F, Karimi N. Experimental investigation of entropy waves' evolution for understanding of indirect combustion noise in gas turbine combustors. Energy 2020;195:116978. https://doi.org/10.1016/j.energy.2020.116978.

[34] Giusti A, Worth NA, Mastorakos E, Dowling AP. Experimental and numerical investigation into the propagation of entropy waves. AIAA J., vol. 55, American Institute of Aeronautics and Astronautics Inc.; 2017, p. 446-58. https://doi.org/10.2514/1.J055199.

[35] Giusti A, Magri L, Zedda M. Flow Inhomogeneities in a Realistic Aeronautical Gas-Turbine Combustor: Formation, Evolution, and Indirect Noise. J Eng Gas Turbines Power 2019;141. https://doi.org/10.1115/1.4040810.

[36] Ron E, Chana K. Comparison of Large Eddy Simulation and Unsteady Reynolds-Averaged Navier-Stokes for Evaluation of Entropy Noise. J Fluids Eng Trans ASME 2017;139. https://doi.org/10.1115/1.4037140.

[37] Gant F, Gruber A, Bothien MR. Development and validation study of a 1D analytical model for the response of reheat flames to entropy waves. Combust Flame 2020;222:305-16. https://doi.org/10.1016/j.combustflame.2020.09.005.

[38] Gant F, Bunkute B, Bothien MR. Reheat flames response to entropy waves. Proc Combust Inst 2020. https://doi.org/10.1016/j.proci.2020.05.007.

[39] Moreau S, Becerril C, Gicquel LYM. Large-Eddy-simulation prediction of indirect combustion noise in the entropy wave generator experiment. Int J Spray Combust Dyn 2018;10:154-68. https://doi.org/10.1177/1756827717740775.

[40] Morgans AS, Goh CS, Dahan JA. The dissipation and shear dispersion of entropy waves in combustor thermoacoustics. J Fluid Mech 2013;733:R2-R2. https://doi.org/10.1017/jfm.2013.448. 
1 [41] Wassmer D, Schuermans B, Paschereit CO, Moeck JP. An Acoustic Time-of-Flight Approach for Unsteady Temperature Measurements: Characterization of Entropy Waves in a Model Gas Turbine Combustor. J Eng Gas Turbines Power 2017;139. https://doi.org/10.1115/1.4034542.

[42] Wassmer D, Schuermans B, Paschereit CO, Moeck JP. Measurement and modeling of the generation and the transport of entropy waves in a model gas turbine combustor. Int J Spray Combust Dyn 2017;9:299-309. https://doi.org/10.1177/1756827717696326.

[43] Hosseinalipour SM, Fattahi A, Khalili H, Tootoonchian F, Karimi N. Experimental investigation of entropy waves' evolution for understanding of indirect combustion noise in gas turbine combustors. Energy 2020;195:116978. https://doi.org/10.1016/j.energy.2020.116978.

[44] Motheau E, Nicoud F, Poinsot T. Mixed acoustic-entropy combustion instabilities in gas turbines. J Fluid Mech 2014;749:542-76. https://doi.org/10.1017/jfm.2014.245.

[45] Bach T, Huet M, Watson R, Spence S, Mahmoudi Y. Effect of Film Cooling on Entropy Noise Generation in a Stator Blade Row. AIAA J 2021.

[46] Persico G, Gaetani P, Spinelli A. Assessment of synthetic entropy waves for indirect combustion noise experiments in gas turbines. Exp Therm Fluid Sci 2017;88:376-88. https://doi.org/10.1016/j.expthermflusci.2017.06.012.

[47] Wang G, Liu X, Wang S, Li L, Qi F. Experimental investigation of entropy waves generated from acoustically excited premixed swirling flame. Combust Flame 2019;204:85-102. https://doi.org/10.1016/j.combustflame.2019.03.005.

[48] Christodoulou L, Karimi N, Cammarano A, Paul M, Navarro-Martinez S. State prediction of an entropy wave advecting through a turbulent channel flow. J Fluid Mech 2020;882. https://doi.org/10.1017/JFM.2019.799.

[49] Sinai YL. The generation of combustion noise by chemical inhomogeneities in steady, low-Mach- 
number duct flows. J Fluid Mech 1980;99:383-97. https://doi.org/10.1017/S0022112080000663.

[50] Magri L, O’Brien J, Ihme M. Compositional inhomogeneities as a source of indirect combustion noise. J Fluid Mech 2016;799. https://doi.org/10.1017/jfm.2016.397.

[51] Magri L, O’Brien J, Ihme M. Effects of nozzle Helmholtz number on indirect combustion noise by compositional perturbations. J Eng Gas Turbines Power 2018;140.

[52] De Domenico F, Rolland EO, Hochgreb S. A generalised model for acoustic and entropic transfer function of nozzles with losses. J Sound Vib 2019;440:212-30. https://doi.org/10.1016/j.jsv.2018.09.011.

[53] De Domenico F, Rolland EO, Rodrigues J, Magri L, Hochgreb S. Compositional and entropy indirect noise generated in subsonic non-isentropic nozzles. J Fluid Mech 2021;910:5. https://doi.org/10.1017/jfm.2020.916.

[54] Rodrigues J, Busseti A, Hochgreb S. Numerical investigation on the generation, mixing andconvection of entropic and compositional waves in a flow duct. J Sound Vib 2020;472:115155. https://doi.org/10.1016/j.jsv.2019.115155.

[55] Mostafa Hosseinalipour S, Rahmani E, Fattahi A, Karimi N. Experimental investigation of the hydrodynamic effects upon convecting entropy waves in nozzle flows. Aerosp Sci Technol 2020;107:106301. https://doi.org/10.1016/j.ast.2020.106301.

[56] Fattahi A, Hosseinalipour SM, Karimi N, Saboohi Z, Ommi F. On the response of a lean-premixed hydrogen combustor to acoustic and dissipative-dispersive entropy waves. Energy 2019;180:27291. https://doi.org/10.1016/j.energy.2019.04.202.

[57] De Domenico F, Rolland EO, Rodrigues J, Magri L, Hochgreb S. Compositional and entropy indirect noise generated in subsonic non-isentropic nozzles. J Fluid Mech 2021;910:5. https://doi.org/10.1017/jfm.2020.916. 
1 [58] Rodrigues J, Busseti A, Hochgreb S. Numerical investigation on the generation, mixing andconvection of entropic and compositional waves in a flow duct. J Sound Vib 2020;472:115155. https://doi.org/10.1016/j.jsv.2019.115155.

[59] Domenico F De, Shah P, Lowe SM, Fan L, Ewart P, Williams BAO, et al. High Frequency Measurement of Temperature and Composition Spots with LITGS. J Eng Gas Turbines Power 2019;141. https://doi.org/10.1115/1.4041275.

[60] Lefebvre AH. Gas turbine combustion. CRC press; 1998.

[61] Nicoud F, Ducros F. Subgrid-scale stress modelling based on the square of the velocity gradient tensor. Flow, Turbul Combust 1999;62:183-200. https://doi.org/10.1023/A:1009995426001.

[62] Lesieur M, Métais O, Comte P. Large-eddy simulations of turbulence. Cambridge university press; 2005.

[63] Smagorinsky J. General circulation experiments with the primitive equations: I. The basic experiment. Mon Weather Rev 1963;91:99-164.

[64] Franke J, Hellsten A, Schlunzen KH, Carissimo B. The COST 732 Best Practice Guideline for CFD simulation of flows in the urban environment: a summary. Int J Environ Pollut 2011;44:41927.

[65] Magri L, O’Brien J, Ihme M. Compositional inhomogeneities as a source of indirect combustion noise. J Fluid Mech 2016;799. https://doi.org/10.1017/jfm.2016.397.

[66] Williams FA. Turbulent combustion. Math. Combust., SIAM; 1985, p. 97-131.

[67] Fattahi A, Karimi N, Hajialigol N. Dynamics of entropy wave generation in a simplified model of gas turbine combustor: A theoretical investigation. Phys Fluids 2020;32:106107. https://doi.org/10.1063/5.0021729. 
1 [68] Job G, Herrmann F. Chemical potential - A quantity in search of recognition. Eur J Phys 2006;27:353-71. https://doi.org/10.1088/0143-0807/27/2/018.

3 [69] Pope SB. Turbulent flows 2001.

4 [70] Celik IB, Cehreli ZN, Yavuz I. Index of resolution quality for large eddy simulations. J. Fluids Eng. Trans. ASME, vol. 127, American Society of Mechanical Engineers Digital Collection; 2005, p. 949-58. https://doi.org/10.1115/1.1990201.

[72] Ries F, Nishad K, Dressler L, Janicka J, Sadiki A. Evaluating large eddy simulation results based

[71] Tavakol MM, Abouali O, Yaghoubi M. Large eddy simulation of turbulent flow around a wall mounted hemisphere. Appl Math Model 2015;39:3596-618. https://doi.org/10.1016/j.apm.2014.11.055. on error analysis. Theor Comput Fluid Dyn 2018;32:733-52. https://doi.org/10.1007/s00162-0180474-0.

[73] Moser RD, Kim J, Mansour NN. Direct numerical simulation of turbulent channel flow up to Re $\tau=590$. Phys Fluids 1999;11:943-5. https://doi.org/10.1063/1.869966.

[74] Shavit G, Lavan Z. Analytical and Experimental Investigations of Laminar Mixing of Confined Heterogeneous Jets. AIAA J 1973;11:352-8. https://doi.org/10.2514/3.50475.

[75] Hajialigol N, Mazaheri K. The dissipation and dispersion of the entropy wave caused by a turbulent lean premixed flame in a combustor. Appl Therm Eng 2020;165:112900. https://doi.org/10.1016/j.applthermaleng.2018.11.019.

[76] Montgomery DC, Runger GC. Applied statistics and probability for engineers. John Wiley \& Sons; 2010.

[77] Lighthill J. Waves in Fluids. Meas Sci Technol 2002;13:1501. https://doi.org/10.1088/09570233/13/9/707. 
1 [78] Mathieu J, Scott J. An Introduction to Turbulent Flow 2000.

2 https://doi.org/10.1017/CBO9781316529850.

3 